\title{
Pre-main sequence spectroscopic binaries suitable for VLTI observations ${ }^{\star} \star \star$
}

\author{
E. W. Guenther ${ }^{1}$, M. Esposito ${ }^{1,2}$, R. Mundt ${ }^{3}$, E. Covino ${ }^{4}$, J. M. Alcalá ${ }^{4}$, F. Cusano ${ }^{1}$, and B. Stecklum ${ }^{1}$ \\ 1 Thüringer Landessternwarte Tautenburg, Sternwarte 5, 07778 Tautenburg, Germany \\ e-mail: guenther@tls-tautenburg.de \\ 2 Dipartimento di Fisica "E.R. Caianiello", Università di Salerno, via S. Allende, 84081 Baronissi (Salerno), Italy \\ 3 Max-Planck-Institut für Astronomie, Königstuhl 17, 69117 Heidelberg, Germany \\ ${ }^{4}$ INAF - Osservatorio Astronomico di Capodimonte via Moiariello 16, 80131 Napoli, Italy
}

Received 24 May 2006 / Accepted 6 February 2007

\begin{abstract}
Context. A severe problem for research in star-formation is that the masses of young stars are almost always estimated from evolutionary tracks alone. Since the tracks published by different groups differ, it is often only possible to give a rough estimate of the masses of young stars. It is thus crucial to test and calibrate the tracks. Up to now, only a few tests of the tracks could be carried out. However, it is now possible with the VLTI to set constrains on the tracks by determining the masses of many young binary stars precisely.

Aims. In order to use the VLTI efficiently, a first step is to find suitable targets, which is the purpose of this work. Given the distance of nearby star-forming regions, suitable VLTI targets are binaries with orbital periods between at least 50 days and a few years. Although a number of surveys for detecting spectroscopic binaries have been carried out, most of the binaries found so far have periods that are too short.

Methods. We thus surveyed the Chamaeleon, Corona Australis, Lupus, Sco-Cen, and $\rho$ Ophiuci star-forming regions in order to search for spectroscopic binaries with periods longer than 50 days, which are suitable for the VLTI observations.

Results. As a result of the 8 year campaign, we discovered 8 binaries with orbital periods longer than 50 days. Amongst the newly discovered long-period binaries is CS Cha, which is one of the few classical T Tauri stars with a circumbinary disk. The survey is limited to objects with masses higher than 0.1 to $0.2 M_{\odot}$ for periods between 1 and 8 years.

Conclusions. We find that the frequency of binaries with orbital periods $\leq 3000$ days is of $20 \pm 5 \%$. The frequency of long and short period pre-main sequence spectroscopic binaries is about the same as for stars in the solar neighbourhood. In total 14 young binaries are now known that are suitable for mass determination with the VLTI.
\end{abstract}

Key words. binaries: spectroscopic - stars: formation - techniques: interferometric - stars: pre-main sequence techniques: radial velocities

\section{Introduction}

The most fundamental parameter of a star is its mass, which determines almost everything about its birth, life, and death. The masses of low-mass pre-main sequence (pms) stars are often derived by comparing the location of the star in the HertzsprungRussell diagram with theoretically calculated evolutionary models. Unfortunately, the evolutionary models published by various authors differ considerably due to the differences in the input physics, such as the treatment of convection, magnetic fields, chemical abundance of the star, etc. (Palla \& Stahler 1992; D’Antona \& Mazzitelli 1994; Swenson et al. 1994; Burrows et al. 1997; Forestini 1994; Siess et al. 1997; Baraffe et al. 1998; Palla \& Stahler 1999; Tout et al. 1999; Chabrier et al. 2000; D’Antona et al. 2000; Wuchterl \& Tscharnuter 2003; Montalbán et al. 2004). Testing and calibrating evolutionary models of

* based on observations obtained at the European Southern Observatory at La Silla, Chile in program 62.I-0418(A); 63.I0096(A); 64.I-0294(A); 65.I-0012(A); 67.C-0155(A); 68.C-0292(A); 68.C-0561(A); 69.C-0207(A); 70.C-0163(A); 073.C-0355(A); 074.A9018(A); 075.C-0399(A-F).

$\star \star$ Tables $2,3,5,6,8,10,12-20$ are only available in electronic form at http://www . aanda.org pms-stars are thus very important for understanding young stars, the determination of the initial mass function, and for studies of the galactic star formation history in general. The lack of knowledge about which models to choose, is thus a severe problem for the whole field of star-formation. Classical T Tauri stars (henceforth called CTTSs) are young, low-mass, optically visible pmsemission line stars with an accretion disk. Weak-line T Tauri stars (WTTSs) are similar to the classical ones but have much lower accretion rates and less massive disks, if any.

An ideal test of the tracks would be to compare the true masses of CTTSs and WTTSs with the masses derived from the models. This is, for example, possible for eclipsing binary stars. Unfortunately, by now there are only three eclipsing young binaries known after eliminating RX J1608.6-3922 by showing that it is not an eclipsing binary but a spotted single star (Joergens et al. 2001). Casey et al. (1998) derived the masses of the eclipsing binary TY CrA to $3.16 \pm 0.02 M_{\odot}$ and $1.64 \pm 0.01 M_{\odot}$ and compared these values with three sets of models. Evolutionary models do not differ too much in the mass range between 1.5 and $3.0 M_{\odot}$, and thus the authors find that all three sets of models are reasonably consistent with the observations. Since many pms-stars have masses lower than $1.5 M_{\odot}$, it is important to also have direct mass determinations in the low-mass domain. It is thus 
better to focus on stars with masses lower than $1.5 M_{\odot}$. Covino et al. (2004) analysed the eclipsing binary RX J 0529.4+0041, which consists of a $1.25 M_{\odot}$ primary star, and a $0.91 M_{\odot}$ companion star and compared these values with three sets of evolutionary models. They find that the models published by Baraffe et al. (1998) and Swenson et al. (1994) are in reasonable agreement with the observations but the ones published by D'Antona \& Mazzitelli (1994) are not. Stassun et al. (2004) analysed the eclipsing binary V1174 Ori, which consists of a $1.01 \pm 0.02 M_{\odot}$ primary and a $0.731 \pm 0.008 M_{\odot}$ secondary. They find that models by Montalbán et al. (2004) seem to agree with the observations, but those published by Siess et al. (1997) conflict with them.

By measuring the orbital motion of the molecular gas in the disk, Simon et al. (2000) conclude that models of the pms evolution with lower $T_{\text {eff }}$ values are in better agreement with the observations (see, e.g., Baraffe et al. 1998; and Palla \& Stahler 1999). Another possibility for measuring masses directly is to combine astrometric data obtained with the HST Fine Guidance Sensors with radial velocity $(R V)$ measurements. In this way Steffen et al. (2001) derived masses of $1.5 \pm 0.2 M_{\odot}$ and $0.81 \pm 0.09 M_{\odot}$ for the binary system NTT $045251+3016$. They find that the Baraffe et al. (1998) models with a mixing-length parameter of $\alpha=1.0$ are closest to the measured primary mass. The models published by D'Antona \& Mazzitelli (1994) are clearly inconsistent with the observations. The first determination of the masses of the components of a young binary star using an interferometer has recently been carried out by Boden et al.(2005). They used the Keck interferometer and determined the masses of the two components of HD $98800 \mathrm{~B}$ to $0.70 \pm 0.06 M_{\odot}$ and $0.58 \pm 0.05 M_{\odot}$. With an age of 8 to $20 \mathrm{Myr}$, it is much older than a typical T Tauri star. The object is still young enough to be used for testing the tracks. A reasonable agreement with the models published by Baraffe (1998) could only be achieved by assuming a rather low metallicity of the star.

While all these data allow a very first test of evolutionary models, more mass determinations are definitely needed, especially of stars of lower masses. Shown in Fig. 1 is $\log \left(L / L_{\odot}\right)$ versus $\log \left(M / M_{\odot}\right)$ for all young stars of which the masses have so far been derived. However, we should keep in mind that the position of young stars in the Hertzsprung-Russell diagram depends on the history of the accretion rate. Thus, short-period, eclipsing binaries might not be ideal for testing the tracks, because the components might have had recent mass-exchange. An example where this could have been the case is RX J1603.8-3938. In this system both components have almost identical masses but one is a factor two brighter than the other (Guenther et al. 2001).

In order to determine the masses of many more pms-stars over a large mass range we have carried out a survey for longperiod binary stars. For single-line spectroscopic binaries (SB1) only the mass-function $f(m)=\frac{m_{2}^{3} \sin ^{3} i}{\left(m_{1}+m_{2}\right)^{2}}$ can be derived using the $R V$-method. If $f(m)$ is combined with the relative astrometric orbit of the two stars (position angle and relative distance of the stars) and if also its parallax is known, the masses of the two stars can be determined. Using this method, we have derived the masses of the two components of the SB1-system EK Dra (König et al. 2005). This system has, however, an age of about $125 \mathrm{Myr}$ and thus, it is not really young. For double-line spectroscopic binaries (SB2), it is possible to measure $m_{1} \sin ^{3} i$ and $m_{2} \sin ^{3} i$. The individual masses of the stars can then be derived if the $R V$-data is combined with the relative astrometric orbit, or if the system is eclipsing.

Unfortunately, the distance to the nearest star-forming regions is so large that binaries that could be resolved with

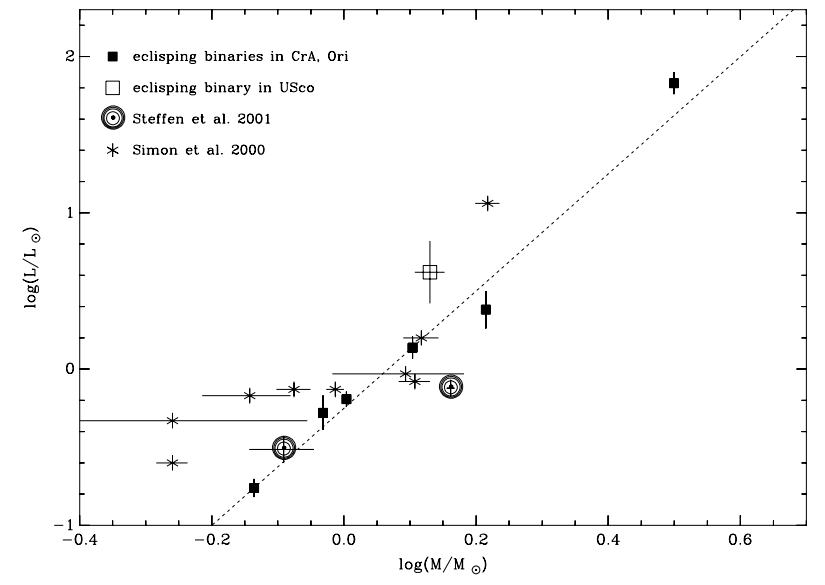

Fig. 1. Current knowledge of empirical mass determination for young stars. The masses of the eclipsing objects in Orion and CrA were taken from Covino et al. (2004), Stassun et al. (2004), and Casey et al. (1998). Alencar et al. (2003) derived the masses of the binary in upper Sco. Using the HST Fine Guidance Sensor, Steffen et al. (2001) determined the masses of NTT 045251+3016 in CrA. Also shown are the masses derived by Simon et al. (2000) by measuring the orbital motion of gas in the disk.

AO-systems on $8 \mathrm{~m}$-class telescopes have orbital periods of many decades, or even centuries. However, the big leap forward in this work is the Very Large Telescope Interferometer, which is now available. The VLTI instrument AMBER (Astronomical Multi BEam combineR) offers the unique opportunity to spatially resolve binaries down to separations of only 4 mas in the $K$-band. Thus, with AMBER it is possible to resolve binaries with separations down to $0.6 \mathrm{AU}$ (corresponding to an orbital period of $\sim 120 \mathrm{~d}$ for systems with $m_{1}=m_{2}=M_{\odot}$ ) for distances $\leq 150 \mathrm{pc}$. Because AMBER works in the near infrared, it is possible to detect a young $0.1 M_{\odot}$ star next to a young $1.0 M_{\odot}$ star due to the achievable brightness-contrast ratio.

To carry out such measurements with AMBER, a sufficient number of pms binaries with suitable separations (i.e. sufficiently long periods) have to be identified first. The aim of this work is thus to identify pms binaries suitable for the VLTI observations. For the survey we selected known pms-stars in southern star-forming regions at a distance $\leq 150 \mathrm{pc}$. Stars fainter than $10 \mathrm{mag}$ in the $K$-band, visual pairs with a separation of $\leq 10$ arcsec, and stars with a $v$ sin $i \geq 50 \mathrm{~km} \mathrm{~s}^{-1}$ are excluded.

Before we started our survey in 1998, only three pmsspectroscopic binaries with orbital periods longer than 50 days located in nearby star-forming regions were known (Mathieu 1994). Four additional spectroscopic binary candidates in the Ophiuchus-Scorpius (Oph), Chamaeleon, Lupus, and Corona Australis star-forming regions were identified by Melo et al. (2003). Thus, while quite a number of authors have already surveyed the nearby star-forming regions for spectroscopic binaries, most of the objects found are binaries with a short period which are unsuitable for VLTI observations. The main intention of our survey is a search for long-period binaries, suitable for VLTI-observations, not a comprehensive survey of spectroscopic binaries. In this paper we present the outcome of this extensive survey. The short-period systems, which were also discovered in the course of the survey, will be subject of a forthcoming paper. In Sect. 2, we describe the observations. In Sect. 3, we give a full list of all long period pms-binaries suitable for the VLTI observations together with the $R V$-values and the orbits of the newly discovered binaries. In Sect. 4, we 
Table 1. Spectroscopic binaries with periods longer than 50 days.

\begin{tabular}{|c|c|c|c|c|c|c|c|c|c|}
\hline & $\begin{array}{l}\text { This survey/ } \\
\text { region }\end{array}$ & $E W \mathrm{H} \alpha$ & $\begin{array}{c}E W \text { LiI } \\
{[\AA]^{1}}\end{array}$ & $\begin{array}{l}\text { Spec type } \\
{[\AA ̊]}\end{array}$ & $\begin{array}{l}m_{K} \\
\text { type }\end{array}$ & $\begin{array}{c}\mathrm{RA} \\
{[\mathrm{mag}]}\end{array}$ & $\begin{array}{l}\text { Dec } \\
(2000.0) \\
\end{array}$ & $\begin{array}{l}\text { Type } \\
(2000.0) \\
\end{array}$ & $\begin{array}{l}\text { Period } \\
\text { [days] }\end{array}$ \\
\hline HIP50796 2 & no / TWA & $0.20^{3}$ & & K5/WTTS & $7.66 \pm 0.03$ & 102218.0 & -103215 & SB1 & 570 \\
\hline CS Cha & yes / Cha & -40 & $0.53 \pm 0.01$ & K4/CTTS & $8.20 \pm 0.03$ & 110226.3 & -773336 & SB1 & $\geq 2482$ \\
\hline HD971314 & no / TWA & & & $\mathrm{F} 2$ & $7.70 \pm 0.02$ & 111034.2 & -302719 & ST3 & 134 \\
\hline RX J-7539 & yes / Cha & fi & $0.21 \pm 0.06$ & K2/WTTS & $7.93 \pm 0.02$ & 122034.4 & -753929 & SB1 & 613 \\
\hline MO Lup 5 & yes / Lup & -2.3 & $0.37 \pm 0.02$ & K7/WTTS & $8.64 \pm 0.02$ & 152403.5 & -320951 & ST3 & $>3000$ \\
\hline RX J1534.1-3916 & yes / Lup & abs & $0.21 \pm 0.02$ & K1/WTTS & $8.55 \pm 0.02$ & 153407.4 & -391618 & SB1 & $>3000$ \\
\hline RXJ1559.2-3814 & yes / Lup & -1.4 & $0.23 / 0.14$ & WTTS & $9.34 \pm 0.03$ & 155916.1 & -381442 & SB2 & 474 \\
\hline GSC 06209-00735 & yes / SC & 0.3 & $0.37 \pm 0.01$ & K2/WTTS & $8.43 \pm 0.02$ & 160814.8 & -190833 & SB1 & 2045 \\
\hline NTTS160814-18576 & no / SC & 0.7 & & K2/WTTS & $7.69 \pm 0.02$ & 161109.0 & -190445 & SB1 & 145 \\
\hline GSC 06213-00306 & yes / SC & fi & $0.24 / 0.18$ & WTTS & $7.43 \pm 0.02$ & 161318.5 & -221248 & SB2 & 167 \\
\hline Haro $1-14 c^{7}$ & no / Oph & & & K3/WTTS & $7.78 \pm 0.03$ & 163104.4 & -240433 & SB2 & 591 \\
\hline NTTS162819-2423s & no / Oph & em & & G8/WTTS & $7.44 \pm 0.02$ & 163120.0 & -243004 & SB1 & 89 \\
\hline BS Indi ${ }^{8}$ & yes / Tuc & abs & $0.18 \pm 0.02$ & K0/WTTS & $6.57 \pm 0.02$ & 212059.8 & -522840 & SB1 & 1222 \\
\hline
\end{tabular}

${ }^{1} \mathrm{em}=$ emission, abs = absorption fi $=$ filled in; ${ }^{2}$ triple system Torres et al. (2003); ${ }^{3}$ Song et al. (2002); ${ }^{4}$ Torres et al. (2003), possibly also a triple system; ${ }^{5}$ triple system consisting of a binary with 12 days, and one of with a period $>3000$ days. (Esposito et al. 2006); ${ }^{6}$ Mathieu (1994); ${ }^{7}$ Reipurth et al. (2002), Simon \& Prato (2004), eccentricity $0.617 \pm 0.008, f(m)=0.018 \pm 0.001 M_{\odot} ;{ }^{8}$ triple system, see Guenther et al. (2005).

discuss the spectroscopic binary candidates, in the last section we discuss the results.

\section{The sample}

For our survey we selected 122 late-type pms-stars in the Chamaeleon (Cha), Corona Australis (CrA), $\rho$ Ophiuchi (Oph), Lupus (Lup), and Scorpius Centaurus (SC) star-forming regions. We did not include the 12 SBs known at the start of the survey (Mathieu et al. 1994; Melo 2003; Guenther et al. 2001), and also generally did not include visual binaries, unless the separation was $\geq 10$ arcsec. After the first observing run, we removed another 14 stars from the list, because their $v \sin i$ turned out to be too high or because these stars are not young. Apart from the known binaries, and stars with very large $v$ sin $i \mathrm{~m}$, we observed essentially all stars with spectral types between F6 and M3 in these regions. The sample that was finally observed comprises 108 stars, of which 26 are CTTS and 82 WTTS.

Determinations of the distance of the Chamaeleon association give values of $160 \pm 15 \mathrm{pc}$ for ChaI, $178 \pm 18 \mathrm{pc}$ for ChaII (Whittet et al. 1997), $171 \pm 20$ pc for ChaI (Wichmann et al. 1998), and $168_{-12}^{+14}$ pc (Bertout et al. 1999). For the Corona Australis region Knude \& Høg (1998) derive a distance of $170 \mathrm{pc}$, which is different from the $\sim 130 \mathrm{pc}$ found by other authors (Knacke et al. 1973; Marraco \& Rydgren 1981). For the $\rho$ Ophiuchi region, the distance of 160 pc derived by Knude \& Høg (1998) agrees with the results obtained by other authors (Whittet 1974; Chini 1981). Quite a number of authors have determined the distance to the Lupus star-forming region: Hughes et al. (1993) find $140 \pm 20$ pc, Knude \& Høg (1998) 100 pc, Nakajima et al. (2000) 150 pc, Sartori et al. (2003) 147 pc, Franco (2002) $150 \mathrm{pc}$, de Zeeuw et al. (1999) $142 \pm 2$ pc, and Teixeira et al. (2000) $85 \mathrm{pc}$, but note that 14 stars in this group have measured parallax-distances, with an average of $138 \mathrm{pc}$.

The Upper Scorpius OB association has an age of 5-6 Myr (Preibisch \& Zinnecker 1999) and thus is slightly older than the other regions that all have ages of 1-3 Myr. The average distance of the Upper Scorpius OB association is $145 \pm 2$ pc (de Zeeuw et al. 1999). In Table 1 we also give the parameters of the binaries found in the TW Hydra (TWA) and Tucana (Tuc) associations. Both regions are older but also closer $(45-60 \mathrm{pc})$ than the other regions. Since low-mass stars in these regions are still pms, these objects are interesting for VLTI observations. The TW Hydra region has an age of 8-12 Myr, and the Tucana association an age of about $30 \mathrm{Myr}$ (Webb et al. 1999; Torres et al. 2000; Torres et al. 2003; Weinberger et al. 2004).

\section{Observations}

All stars were observed with the ESO Echelle spectrograph FEROS (Fiber-fed Extended Range Optical Spectrograph). FEROS was operated up to October 2002 (HJD 245 2550) at the ESO 1.5-m-telescope, and was then moved to the MPG/ESO2.20-m-telescope. The spectra cover the wavelength region between about $3600 \AA$ and $9200 \AA$ with a resolving power of $\lambda / \Delta \lambda=48000$. On the 1.5 -m-telescope the entrance aperture of the fibre had a projected diameter of 2.7 on the sky, while at MPG/ESO 2.2-m-telescope 2"'0. As long as FEROS was at the ESO 1.5-m-telescope, exposure-times were set so that an $S / N$-ratio of about 30 was achieved. The exposuretimes were thus between 5 and $45 \mathrm{~min}$. Since the performance of FEROS dramatically improved when FEROS was moved to the MPG/ESO 2.2-m-telescope, the $S / N$-ratio went up to typically 50, although we shortened the exposure times. The standard FEROS data-reduction pipeline was used for bias subtraction, flat-fielding, scattered light removal, Echelle order extraction, and wavelength calibration of the spectra.

When measuring the radial velocity of stars, the question arises as to whether it is better to use several templates of different spectral types, or only one template. We tried out both methods using HR3862 (G0), HR6748 (G5), HR 5777 (K1), HR5568 (K4), and HR6056 (M0.5) as templates. The advantage in using several templates is that the match between the template and the star is better, but the disadvantage is that the absolute $R V \mathrm{~s}$ of the templates have to be known to a high accuracy, if the absolute $R V \mathrm{~s}$ of a stars are of interest. If the absolute $R V \mathrm{~s}$ of the templates are not well known to a high accuracy, the absolute values of the $R V \mathrm{~s}$ of the stars are lost. In our case this would be a disadvantage, because the first step for detecting pms-spectroscopic binaries is to determine their absolute $R V$. A star with an $R V$ that differs by more than $2 \mathrm{~km} \mathrm{~s}^{-1}$ from that of the star-forming region is likely to be either not a member of that region or an SB1. 


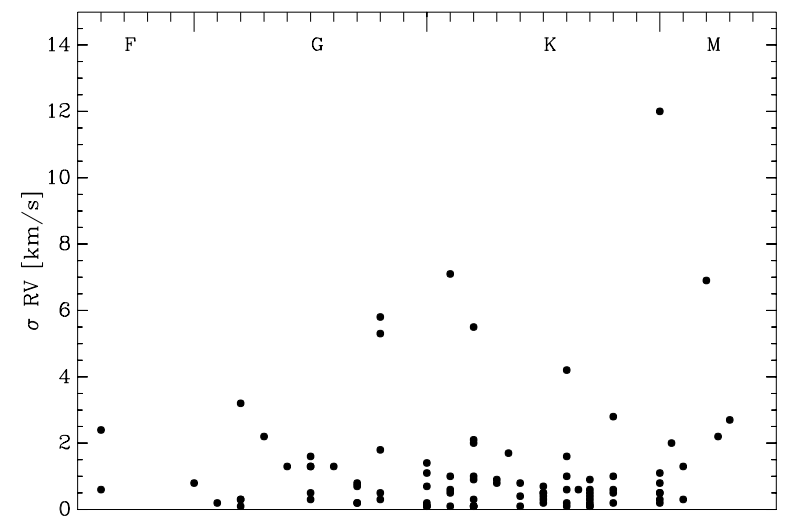

SPEC TYPE

Fig. 2. Scatter of the measurements of all single stars listed in Tables 18-20 versus the spectral type.

The other alternative is to use just one template. This approach is used in the HARPS search for extra-solar planets of F, G, and K-stars, where an accuracy of typically $1 \mathrm{~m} \mathrm{~s}^{-1}$ is achieved (Lovis et al. 2005). A good choice in our case is the K1-star HR 5777, which is bright $\left(m_{v}=4.6\right)$, and its absolute $R V$ is well-determined $\left(+49.12 \pm 0.06 \mathrm{~km} \mathrm{~s}^{-1}\right.$, Murdoch et al. 1993). If we use just one template, the question is whether the mismatch of the spectral type between the template and the star reduces the accuracy of the $R V$-measurements. If this were the case, the accuracy of the $R V$-measurements of the $\mathrm{K}$-stars would be higher than those of stars with other spectral types. Figure 2 shows the variance of the $R V$-measurements versus the spectral type. Assuming that the scatter is the error of the measurements, we conclude that the error dominated by the $v \sin i$ of the stars, and there is no obvious trend with the spectral type. This is possibly because we have only very few M-stars in our survey, where the mismatch with the template really matters. We thus use only HR 5777 as a template. In this way, it is possible to compare the $R V \mathrm{~s}$ of different stars, and it is possible to merge our data with data taken with other instruments.

We split up the spectrum into six wavelength regions which are practically free of stellar emission lines, and virtually free of telluric lines. The wavelength regions are: 4000 to $4850 \AA, 4900$ to $5850 \AA, 5900$ to $6500 \AA$, 6600 to $6860 \AA, 7400$ to $7500 \AA$, and 7700 to $8100 \AA$. For each of the wavelength-band, we obtained the $R V$ separately and then averaged these 6 values. The errors in the $R V$-measurements of the T Tauri stars are determined from the variance of the $R V$-values of the individual spectral regions. In many stars, we could use only the first three regions, because the $S / N$ ratio of the other regions was too low. In such cases we always used the same regions for the same star. In order to account for any instrumental shift, we measured the position of the telluric spectral lines in the 6860 to $6930 \AA$ region by cross-correlating this part of the stellar spectrum with one taken with the Fourier Transform Spectrometer of the McMathPierce telescope at Kitt Peak (Wallace et al. 1998; Brault 1978). We find an instrumental shift of typically $0.5 \mathrm{~km} \mathrm{~s}^{-1}$, which differs typically by less than $0.1 \mathrm{~km} \mathrm{~s}^{-1}$ from frame to frame.

In order to investigate the accuracy of the $R V$-measurements obtained, we took 31 spectra of HR 5777 spread out over the whole observing campaign. These spectra were reduced in the same way as the spectra of our targets. That is, we used one spectrum of HR 5777 as the template (taken on the March 23, 1999), and cross-correlated this spectrum with all other

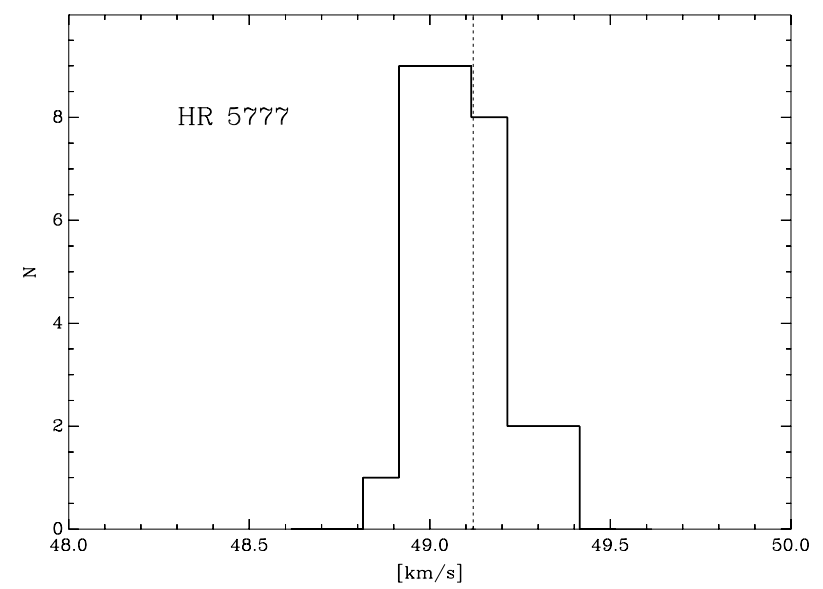

Fig. 3. Histogram of the $R V$ measurements obtained for HR 5777. The published $R V$ of this star is $+49.12 \pm 0.06 \mathrm{~km} \mathrm{~s}^{-1}$ (Murdoch 1993), which is shown as a dashed line. The FEROS measurements are consistent with this value. The $F W H M$ of a Gaussian-fitted to this the distribution is $0.30 \mathrm{~km} \mathrm{~s}^{-1}$. The error of the measurements thus is $\pm 0.15 \mathrm{~km} \mathrm{~s}^{-1}$.

spectra taken of HR 5777. The absolute $R V$ of the template was determined by cross-correlating it with the FTS-spectrum of the sun, which was rebinned to the same resolution as the FEROS spectra (Wallace et al. 1998; Brault 1978). Figure 3 shows a histogram of the $R V$-measurements of HR 5777. The $R V$-values determined for HR 5777 are perfectly consistent with the published $R V$ of $+49.12 \pm 0.06 \mathrm{~km} \mathrm{~s}^{-1}$ (Murdoch et al. 1993). Fitting a Gaussian to this distribution, we derived an FWHM of $0.30 \mathrm{~km} \mathrm{~s}^{-1}$. This implies that the error is $\pm 0.15 \mathrm{~km} \mathrm{~s}^{-1}$, which agrees well with the error derived from the variance of the $R V$-values, which is $\pm 0.12 \mathrm{~km} \mathrm{~s}^{-1}$. We thus take $\pm 0.15 \mathrm{~km} \mathrm{~s}^{-1}$ as the intrinsic uncertainty of the measurements. The uncertainty of the $R V$-measurements of the pre-main sequence stars, as determined from the variance of the $R V$-measurements of the individual spectral regions, are usually larger than those of HR 5777 , because of the smaller signal-to-noise and the larger $v \sin i$ of the stars.

\section{Results: long-period spectroscopic binaries}

The aim of this survey is to detect young binaries suitable for VLTI-observations, i.e. binaries with orbital periods longer than 50 days located in the nearby star-forming regions. While in our optical observations these systems usually appear as SB1s, many of these can be converted into SB2s, if high-resolution infrared spectra were taken (e.g. Prato et al. 2002). Table 1 gives a short overview of all known long-period binaries in nearby southern star-forming regions found during this study (CS Cha, RX J-7539, MO Lup, RX J1534.1-3916, RX J1559.23814, GSC 06209-00735, GSC 06213-00306, BS Indi) and by other authors. In the following, we give details on the individual systems listed in Table 1 . In addition to the binaries found in this survey, we also list in Table 1 the long-period spectroscopic binaries published elsewhere (HIP50796, Torres et al. 2003; HD97131, Torres et al. 2003; NTTS160814-1857, Mathieu 1994; Haro 1-14c, Reipurth et al. 2002, Simon \& Prato 2004; NTTS162819-2423s, Mathieu 1994). While we cannot completely rule out that the VW Cha and GSC 06793-00569 are binaries, these objects are in any case unsuitable for the VLTI observations, and thus not listed in Table 1. 


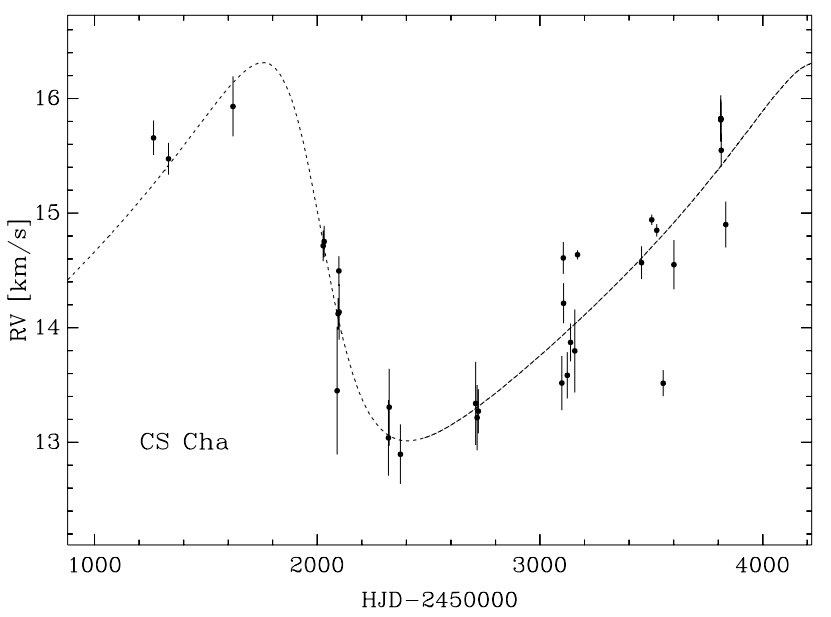

Fig. 4. The $R V$-measurements of the CTTS CS Cha, together with a possible orbital solution with a period of 2482 days.

\subsection{CS Cha}

CS Cha is a CTTS of solar abundance (Padgett 1996). This object was observed by Ghez et al. (1997) by means of speckleimaging, but no companion was found within 0.1 . However, Takami et al. (2003) already conclude based on a large gap in the inner disk, that this object might actually be a binary. The basic properties of the disk of this star were derived by Henning et al. (1993). We took 32 spectra of this object over a period of 2570 days (Table 2). CS Cha is an SB1-binary system with a very long period. A possible orbit of 2482 days is shown, together with the $R V$-measurements in Fig. 4 . The minimum mass of the companion in this case would be only about $0.1 M_{\odot}$. However, it is still possible that the orbital period is even longer than 2482 days.

\section{2. $R X J 1220.6-7539$}

RX J1220.6-7539 is a WTTS. In most of our spectra, the $\mathrm{H} \alpha$ line is filled in. In some occasions we observed a small double-line emission profile with an equivalent width of only $-0.4 \AA$. On other occasions, $\mathrm{H} \alpha$ appears in absorption but the equivalent width then is only $0.08 \AA$. We took 33 spectra of this star over a time-span of 2587 days. The object is an SB1-binary with an orbital period of $613.9 \pm 0.4$ days and an eccentricity of $0.225 \pm$ 0.005 . Figure 5 shows the phase-folded $R V$-measurements. The $R V$-measurements are given in Table 3 , and the orbital elements in Table 4.

\section{3. $R X J 1534.1-3916$}

RX J1534.1-3916 is a WTTS, with $\mathrm{H} \alpha$ in absorption. We took 24 spectra of this star over a time-span of 2574 days. The $R V$-values are shown in Table 5 and Fig. 6. The orbital period is certainly much longer than the 2574 days over which we observed the star. Thus we cannot derive an orbit yet. The eccentricity has to be very high $(\approx 0.9)$. RX J1534.1-3916 nicely illustrates the difficulty in finding long-period spectroscopic binaries with eccentric orbits. If we had not taken the first two spectra, we probably would have classified this object as a single star. According to Hogeveen (1992), only $0.45 \%$ of the binaries have $e \geq 0.9$.
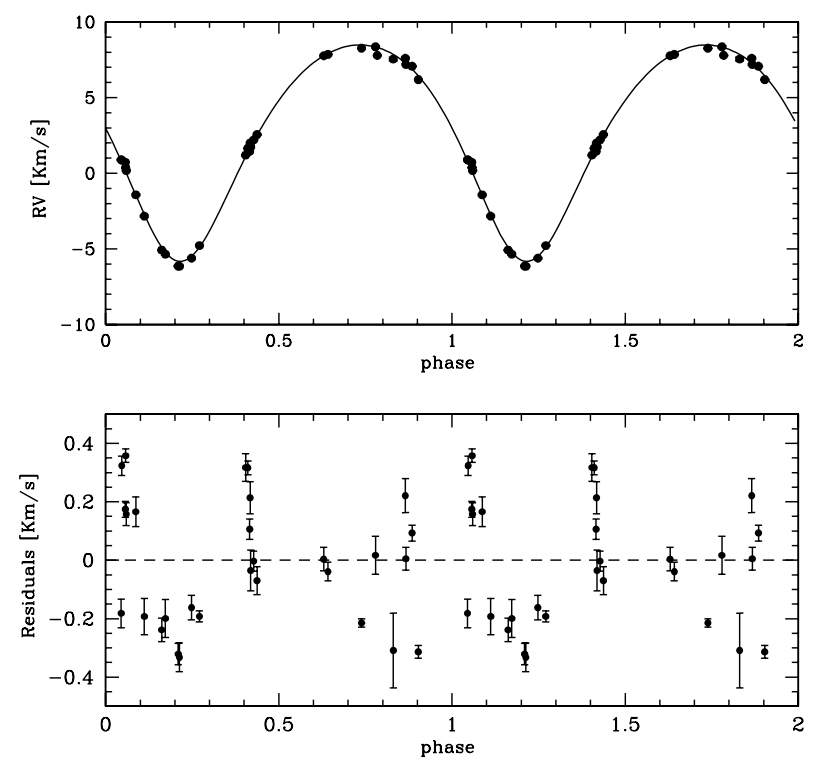

Fig. 5. The phase-folded $R V$-measurements of RXJ1220.6-7539. The orbital period is $612.9 \pm 0.4$ days.

Table 4. Orbital elements of RX J1220.6-7539.

\begin{tabular}{ll}
\hline \hline Element & Value \\
\hline$P$ & $613.9 \pm 0.4 \mathrm{~d}$ \\
$T_{0}[\mathrm{HJD}]$ & $2450123 \pm 3$ \\
$\gamma$ & $2.74 \pm 0.03 \mathrm{~km} \mathrm{~s}^{-1}$ \\
$K_{1}$ & $7.15 \pm 0.07 \mathrm{~km} \mathrm{~s}^{-1}$ \\
$e$ & $0.225 \pm 0.005$ \\
$\omega$ & $171.7 \pm 1.9^{\circ}$ \\
$a_{1} \sin i$ & $0.399 \pm 0.005 \mathrm{AU}$ \\
$f(m)$ & $0.0228 \pm 0.0008 M_{\odot}$ \\
\hline
\end{tabular}

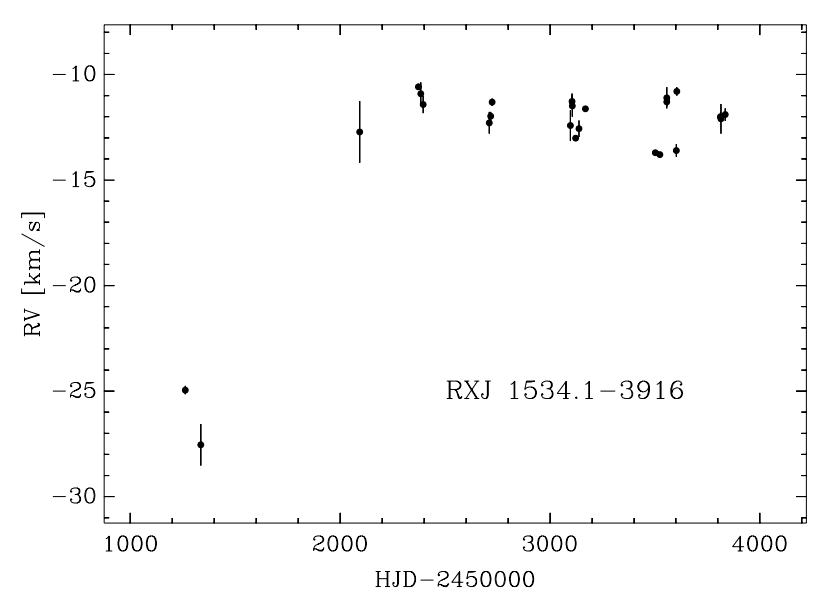

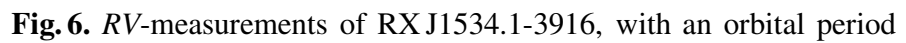
that is apparently longer than 7 years.

\section{4. $R X J 1559.2-3814$}

RX J1559.2-3814 is a WTTS and an SB2. The average equivalent width of $\mathrm{H} \alpha$ is $-1.4 \pm 0.2 \AA$. The equivalent width of the LiI $6708 \AA$ line is $0.23 \AA$ for the primary and $0.14 \AA$ for the secondary component. We find that the ratio of the height of the peak of the cross-correlation function for the two components is $1.9 \pm 0.2$. Table 6 gives the $R V$ measurements obtained for both components, and the orbital elements are listed in the Table 7. A good fit is obtained for a period of 474 days (Fig. 7). 
Table 7. Orbital elements RX J1559.2-3814.

\begin{tabular}{ll}
\hline \hline Element & Value \\
\hline$P$ & $474.0 \pm 1.0 \mathrm{~d}$ \\
$T_{0}[\mathrm{HJD}]$ & $2447834 \pm 3$ \\
$\gamma$ & $2.0 \pm 0.4 \mathrm{~km} \mathrm{~s}^{-1}$ \\
$K_{1}$ & $13.4 \pm 0.2 \mathrm{~km} \mathrm{~s}^{-1}$ \\
$K_{2}$ & $14.2 \pm 0.2 \mathrm{~km} \mathrm{~s}^{-1}$ \\
$e$ & $0.336 \pm 0.005$ \\
$\omega_{1}$ & $336.8 \pm 1.9^{\circ}$ \\
$\omega_{2}$ & $156.8 \pm 1.9^{\circ}$ \\
$a_{1} \sin i$ & $0.549 \pm 0.010 \mathrm{AU}$ \\
$a_{2} \sin i$ & $0.581 \pm 0.010 \mathrm{AU}$ \\
$q=\frac{m_{2}}{m_{1}}$ & $0.945 \pm 0.027$ \\
$m_{1} \sin ^{3} i$ & $0.444 \pm 0.026 M_{\odot}$ \\
$m_{2} \sin ^{3} i$ & $0.419 \pm 0.025 M_{\odot}$ \\
\hline &
\end{tabular}
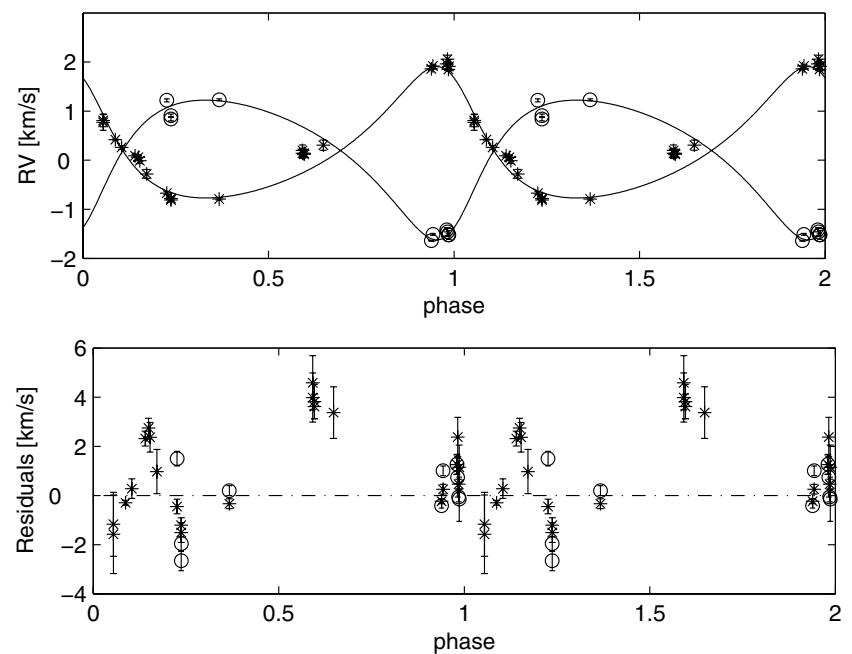

Fig. 7. $R V$-measurements of $\mathrm{RXJ} 1559.2-3814$. Shown is the orbit of the $\mathrm{A}$ and the $\mathrm{B}$ component for a period of 474 days.

\subsection{GSC 06209-00735}

GSC 06209-00735 was first identified as a member of the Upper Sco association based on the spectroscopic and X-ray properties by Preibisch et al. (1998). We confirm the large equivalent width of the LiI $6708 \AA$ line of $0.37 \pm 0.01 \AA$, and found that the object is an $\mathrm{SB} 1 . \mathrm{H} \alpha$ is in absorption, and the equivalent width is $0.3 \pm$ $0.1 \AA$. In total, we took 26 spectra within 2287 days. We find an orbital period of $2045 \pm 16$ days, which is only slightly lower than the time over which we observed the star. Figure 8 shows the phase-folded $R V$-measurements together with the orbit. The $R V$-measurements are given in Table 8, and the orbital elements in Table 9.

\subsection{GSC 06213-00306}

GSC 06213-00306 is another SB2 WTTS. The equivalent widths of the Li I $6708 \AA$ line are $0.241 \pm 0.004 \AA$ and $0.18 \pm 0.04 \AA$ for the primary and secondary, respectively. The $\mathrm{H} \alpha$-line is often filled in, and occasionally it appears as an emission line with an equivalent width of $-0.1 \AA$, on other occasions as an absorption line with an equivalent width of $0.1 \AA$. In total, we took 22 spectra of this binary. We find an orbital solution with a period of $166.9 \pm 0.1$ days. Thus, GSC 06213-00306 is a long-period, young SB2-binary, the orbital elements for both components have been solved. Given that it has a $K$-magnitude of $7.43 \pm 0.02$
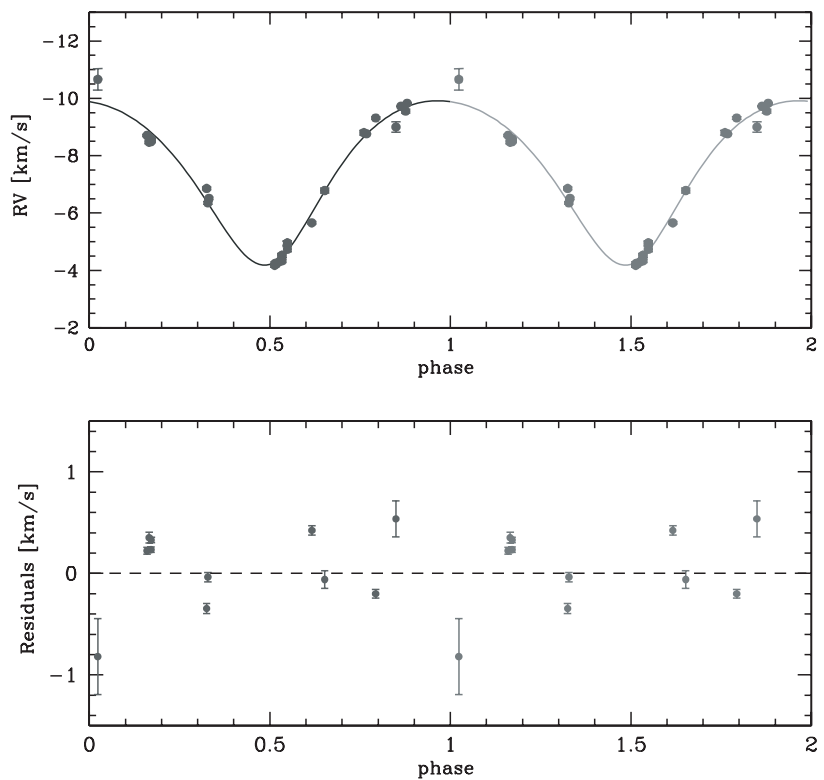

Fig. 8. Phase-folded $R V$-measurements of GSC 06209-00735, with an orbital period of $2045 \pm 16$ days.

Table 9. Orbital elements of GSC 06209-00735.

\begin{tabular}{ll}
\hline \hline Element & Value \\
\hline$P$ & $2045 \pm 16 \mathrm{~d}$ \\
$T_{0}[\mathrm{HJD}]$ & $2451022 \pm 12$ \\
$\gamma$ & $-7.62 \pm 0.02 \mathrm{~km} \mathrm{~s}^{-1}$ \\
$K_{1}$ & $2.87 \pm 0.05 \mathrm{~km} \mathrm{~s}^{-1}$ \\
$e$ & $0.20 \pm 0.03$ \\
$\omega$ & $8.1 \pm 2.3^{\circ}$ \\
$a_{1} \sin i$ & $0.53 \pm 0.018 \mathrm{AU}$ \\
$\mathrm{f}(\mathrm{m})$ & $0.0049 \pm 0.0005 M_{\odot}$ \\
\hline
\end{tabular}

Table 11. Orbital elements GSC 06213-00306.

\begin{tabular}{ll}
\hline \hline Element & Value \\
\hline$P$ & $166.9 \pm 0.1 \mathrm{~d}$ \\
$T_{0}[\mathrm{HJD}]$ & $2452124.2 \pm 1.1$ \\
$\gamma$ & $-6.76 \pm 0.06 \mathrm{~km} \mathrm{~s}^{-1}$ \\
$K_{1}$ & $15.1 \pm 0.1 \mathrm{~km} \mathrm{~s}^{-1}$ \\
$K_{2}$ & $15.65 \pm 0.09 \mathrm{~km} \mathrm{~s}^{-1}$ \\
$e$ & $0.226 \pm 0.006$ \\
$\omega_{1}$ & $161.8 \pm 2.2^{\circ}$ \\
$\omega_{2}$ & $341.8 \pm 2.2^{\circ}$ \\
$a_{1} \sin i$ & $0.226 \pm 0.002 \mathrm{AU}$ \\
$a_{2} \sin i$ & $0.234 \pm 0.002 \mathrm{AU}$ \\
$q=\frac{m_{2}}{m_{1}}$ & $0.97 \pm 0.01$ \\
$m_{1} \sin ^{3} i$ & $0.246 \pm 0.006 M_{\odot}$ \\
$m_{2} \sin ^{3} i$ & $0.239 \pm 0.006 M_{\odot}$ \\
\hline
\end{tabular}

it is an ideal VLTI target. The $R V$-measurements are listed in Table 10, the orbit in Table 11. The $R V$-measurements and the orbit are shown in Fig. 9. It is interesting to note that the masses of both components are almost identical.

\section{Results: spectroscopic binary candidates}

In this section we discuss two objects that were suspected to be spectroscopic binaries with a long period. We argue that in both cases it is unlikely that these are spectroscopic binaries. 

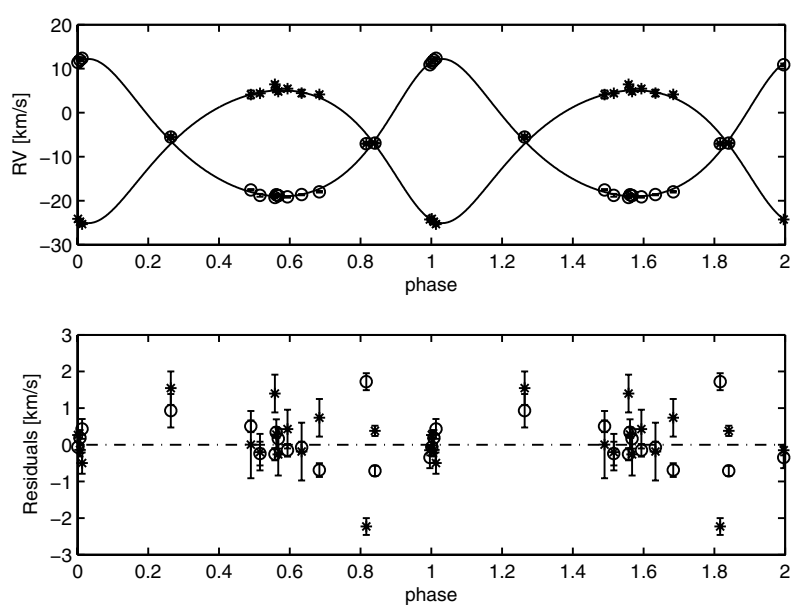

Fig. 9. Phase-folded $R V$-measurements of GSC 06213-00306, together with the residuals. The orbital period is $166.87 \pm 0.13$ days.

\subsection{VW Cha}

VW Cha was already been observed by Melo (2003) with FEROS, who found an occasional doubling of the crosscorrelation peak and thus suggested that this object is a spectroscopic binary. Brandner et al. (1996) find that VW Cha is a visual binary with a separation of $0.72 \pm 00^{\prime} 03$. The companion is only $0.25 \mathrm{mag}$ fainter in $J$ than the primary. Using $K$-band speckle observations, Ghez et al. (1997) confirm the presence

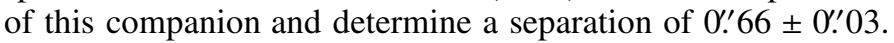
Surprisingly, Ghez et al. (1997) give a flux-ratio in the $K$-band of $4.5 \pm 0.7$. In addition to this inner component, Ghez et al. (1997) detected another component with a separation of $17 \pm 2^{\prime \prime}$.

As pointed out above, FEROS was operated up to October of 2002 (HJD 2452550) at the ESO 1.5-m telescope and was then moved to the MPG/ESO-2.20-m telescope. The entrance apertures were 2.77 and $2 . \prime 0$ on the ESO 1.5-m telescope, and the MPG/ESO 2.2-m telescope, respectively. Thus, if the fibre is centred on the primary, the secondary would almost be at the edge. The amount of light from the primary and secondary thus would vary depending on the exact placement of the fibre and the seeing. The question thus arises whether the occasional doubling of the spectral-lines is due to this effect, or whether there is really another component. If VW Cha were a spectroscopic binary, its separation would have to be much smaller than $0.72 \pm 0.0^{\prime} 03$, which implies that we should observe large $R V$-variations.

In order to clarify the situation, we took 16 spectra of this star. The cross-correlation peak is in fact asymmetric. In contrast to our expectation, we find only rather modest variations of the $R V$ (Table 12). Additionally, the $R V$-measurements are erratic, and not periodic. While we cannot completely rule out that this is an SB2, it seems more likely that the asymmetric form of the cross-correlation is due to the visual companion moving in and out of the fibre and not due to a spectroscopic companion. This hypothesis would also explain the observed scatter of the $R V$-measurements, because the velocity difference of two stars of one solar-mass are in a circular orbit with a separation of 0.7 is about $4 \mathrm{~km} \mathrm{~s}^{-1}$. This object is, in any case, unsuitable for VLTI-observations.

\subsection{GSC 06793-00569}

GSC 06793-00569 is a WTTS with a weak H $\alpha$-emission line of $-0.4 \AA$. We took 19 spectra of this star. Although the

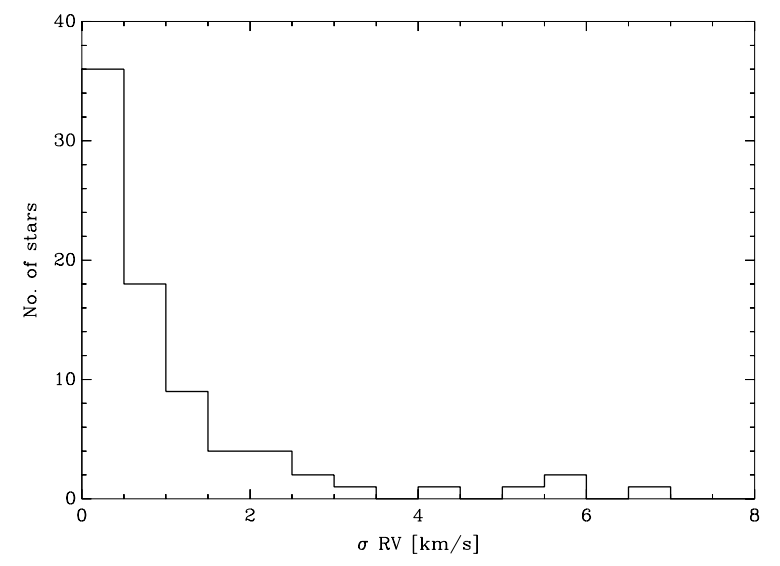

Fig. 10. Histogram of the $R V$-variations observed for the stars listed in Tables $18-20$. The amplitude of the $R V$-variations caused are typically less than $3 \mathrm{~km} \mathrm{~s}^{-1}$.

cross-correlation occasionally shows a blue asymmetry, and on other occasions a red asymmetry, the $R V$-variations are moderate, and we do not find any significant periodicity (Table 13). We thus interpret the data in the same way as for VW Cha, the star could be a visual binary with such a separation that roughly corresponds to the radius of the fibre.

\section{Results: single stars}

Unfortunately, not all previous surveys for pms-binaries give a list of the objects that were found to be single. Such a list is, however, very useful, because it avoids duplication of work. In Tables 18-20 we list the average $R V$-values obtained for all single stars together with the variance of the $R V$-values determined for each star. Also given in these tables are the average equivalent widths of $\mathrm{H} \alpha$ and the $\mathrm{Li}$ I $6708 \AA$ line, as derived from the spectra. The $K$-band brightness is taken from the 2MASS AllSky Catalogue of Point Sources (Cutri et al. 2003). The number of spectra taken for each star is given in the last column.

The $R V$-curve of a star with a spot is periodic, where the period corresponds to the rotation period of the star. However, once this spot vanishes and other spots appear at different longitudes, the amplitude and the phase of the $R V$-signal changes. As a result, the periodic signal vanishes, and the power in a periodogram decreases. In contrast to this, the signal of an orbiting companion remains unchaged and thus the power in a periodogram always increases, if more data is added. The difference between the $R V$-signal caused by activity and by an orbiting companion is nicely illustrated for EK Dra, $\epsilon$ Eri, and $\beta$ Gem. EK Dra is an active binary star with an orbital period of 45 years, $\epsilon$ Eri is an active star with a planet, and $\beta$ Gem an oscillating star with a planet (König et al. 2005; Hatzes et al. 2000; Hatzes et al. 2006). For more information on the effects of stellar activity, see also Paulson et al. (2002), and Saar et al. (1998). Variations in the $R V$ due to stellar activity have already been observed in $T$ Tauri stars. Because of the activity level of these stars, the effects are even stronger than for older stars. RU Lup, for example, shows $R V$-variations with an amplitude of $2.17 \mathrm{~km} \mathrm{~s}^{-1}$ (Stempels et al. 2007). It is thus not surpising that we observe the same effects in the T Tauri stars that we observed. Nice examples are RX J1415.0-7822 (Table 14, HK Lup, Table 15, GSC 0679301406 Table 16), and GSC 06793-00994 (Fig. 11), of which we took 13 to 19 spectra each. All of these stars show $R V$-variations with a semi-amplitude of 1 to $3 \mathrm{~km} \mathrm{~s}^{-1}$, but in none of them could 


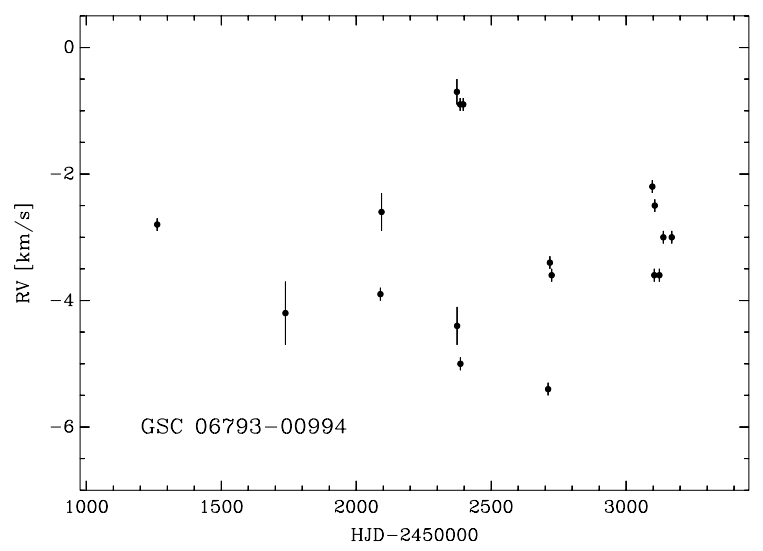

Fig. 11. A typical example of the non-periodic variations of a young star. Shown are the $R V$-values of GSC 06793-00994.

a significant period be detected. Figure 10 shows a histogram of the $R V$-variations observed in single stars.

The survey is limited to companions that cause an $R V$-amplitude of $\geq 3 \mathrm{~km} \mathrm{~s}^{-1}$. For example, a system consisting of a $1.0 M_{\odot}$ primary and a $0.1 M_{\odot}$ secondary would only be detectable, if the period were equal, or shorter, than a year. Similarly, if the companion would have an orbital period of 3000 days, the mass of the companion had to be $\geq 0.23 M_{\odot}$ in order to be detectable. The survey thus is incomplete for longperiod companions with masses $\leq 0.2 M_{\odot}$.

We took 13 to 21 spectra of all those stars that show unusually large $R V$-variations, in order to find out whether these are caused by stellar activity or by an orbiting companion. Apart from the objects listed in Table 1, none of them turned out to be a binary. Only in the case of the two rapidly rotating stars GSC 06781-01046 and MN Lup, we cannot fully exclude that they are binaries. One of the spectra of GSC 06781-01046 shows a double-line appearance. This object thus might be an SB2, but more spectra are needed.

\section{Discussion and conclusions}

The goal of this survey was to compile a list of young longperiod binary stars suitable for calibrating the evolutionary tracks by measuring the masses by combining spectroscopic and VLTI data. Although it was not our intention to carry out a statistically complete survey, it does contain some useful informtion on the frequency of young binaries. In his classical review article Mathieu (1994) listed only 3 pms-spectroscopic binaries located in the nearby southern star-forming regions (Chamaeleon, Corona Australis, Lupus, Sco-Cen, $\rho$ Ophiuchi) with orbital periods longer than 50 days and 4 short period systems in these regions. Another short-period binary system (RX J1108.8-7519) was found by Covino et al. (1997). The long-period binaries are NTTS162819-2423S (period 89.1 days, $\rho$ Oph), Wa Oph 1 (=NTTS160814-1857, period 144.7 days, SC), and Haro 1-14c (period 591 days, $\rho$ Oph) (see also Reipurth et al. 2002). NTTS162819-2423S and Haro 1-14c are both members of hierarchical systems. The distance between the spectroscopic binary Haro 1-14c and Haro 1-14 is 12'.'9, and NTTS162819-2423S is separated from the spectroscopic binary NTTS162819-2423N by 6.'. In addition to these stars, Melo (2003) identified VW Cha (ChaI), BF Cha (ChaII), CHX 18N (ChaI), and AS 205 (SC) as spectroscopic binaries but did not derive the orbital periods of these objects. However, we do not regard VW Cha as a spectroscopic binary. Another spectroscopic binary found is
RX J1603.8-3938 (Guenther et al. 2001). Thus, in all previous surveys, 12 SBs were found: 6 have periods longer than 50 days, and two are triple stars. To these we add 7 long-period SBs and 3 short-period ones. These short-period systems will be discussed in a forthcoming paper (Covino et al. 2007). One of the newly-found long-period systems is a triple star. The newly found triple system is hierarchical. It consists of two components that are separated by $0.26 \pm 0.03$, where one of these components is a binary with an orbital period of possibly 16 days. If we count the triple star as two binaries, because it consists of a long and a short period system, the total number of SBs in these regions is 25 . By adding in the previously known binaries, the sample comprises 120 stars in total. Thus, the frequency of SBs is $20 \pm 5 \%$. This number can now be compared with the results by Duquennoy \& Mayor (1991), who found a frequency of binaries with periods less than 3000 days amongst old G-dwarfs in the solar neighbourhood of $21 \pm 4 \%$. The frequency of old and young binaries is thus roughly the same.

Acknowledgements. We are grateful to the user support group of ESO/La Silla. E.C. and J.M.A. acknowledge financial support from INAF and Italian MIUR. This research has made use of the SIMBAD database, operated at CDS, Strasbourg, France. This publication makes use of data products from the Two Micron All Sky Survey, which is a joint project of the University of Massachusetts and the Infrared Processing and Analysis Center/California Institute of Technology, funded by the National Aeronautics and Space Administration and the National Science Foundation. The authors would also like to thank Andrea Mehner for critically reading the text, and the anonymous referee for improving it.

\section{References}

Alencar, S. H. P., Melo, C. H. F., Dullemond, C. P., et al. 2003, A\&A, 409, 1037 Baraffe, I., Chabrier, G., Allard, F., \& Hauschildt, P. H. 1998, A\&A, 337, 403 Bertout, C., Robichon, N., \& Arenou, F. 1999, A\&A, 352, 574

Beuzit, J. L., Hubin, N., Gendron, E., et al. 1994, in Adaptive Optics in Astronomy, ed. M. A. Ealey, \& F. Merkle, Proc. SPIE, 2201, 955

Boden, A. F., Sargent, A. I., Akeson, R. L., et al. 2005, ApJ, 635, 442

Brault, J. W. 1978, in Proceedings of JOSO Workshop, ed. G. Godoli, G. Noci, \& A. Righini, Arcetri: Osser. Mem. Osserv. Astrofis., 106, 33

Brandner, W., Alcalá, J. M., Kunkel, M., Moneti, A., \& Zinnecker, H. 1996, A\&A, 307, 121

Burrows, A., Marley, M., Hubbard, W.B., et al. 1997, ApJ, 491, 856

Casey, B. W., Mathieu, R. D., Vaz, L. P. R., Andersen, J., \& Suntzeff, N. B. 1998, AJ, 115, 1617

Chabrier, G., \& Baraffe, I. 2000, ARA\&A, 38, 337

Chini, G. 1981, A\&A, 99, 346

Covino, E., Alcala, J. M., Allain, S., et al. 1997, A\&A, 328, 187

Covino, E., Frasca, A., Alcalá, J. M., Paladino, R., \& Sterzik, M. F. 2004, A\&A, 427,637

Covino, E., et al. in preparation

Cutri, R. M., et al. 2003, VizieR Online Data Catalog: II/246. Originally published in: University of Massachusetts and Infrared Processing and Analysis Center, (IPAC/California Institute of Technology)

D’Antona, F., \& Mazzitelli, I. 1994, ApJS, 90, 467

D’Antona, F., Ventura, P., \& Mazzitelli, I. 2000, ApJ, 543, L77

de Zeeuw, P. T., Hoogerwerf, R., de Bruijne, J. H. J., Brown, A. G. A., \& Blaauw, A. 1999, AJ, 117,354

Duquennoy, A., \& Mayor, M. 1991, A\&A, 248, 485

Esposito, M., Covino, E., Alcalá, J. M., Guenther, E. W., \& Schisano, E. 2006, MNRAS, submitted

Forestini, M. 1994, A\&A, 285, 473

Franco, G. A. P. 2002, MNRAS, 331, 474

Ghez, A. M., Neugebauer, G., \& Matthews, K. 1993, AJ, 106, 2005

Ghez, A. M., McCarthy, D. W., Patience, J. L., \& Beck, T. L. 1997, ApJ, 481, 378

Guenther, E. W., Torres, G., Batalha, N., et al. 2001, A\&A, 366, 965

Guenther, E. W., Covino, E., Alcalá, J. M., Esposito, M., \& Mundt, R. 2005, A\&A, 433, 629

Hatzes, A. P., Cochran, W. D., McArthur, B., et al. 2000, ApJ, 544, L145

Hatzes, A. P., Cochran, W. D., Endl, M., et al. 2006, A\&A, 457, 335

Henning, T., Pfau, W., Zinnecker, H., \& Prusti, T. 1993, A\&A, 276, 129

Hogeveen, S. J. 1992, Ap\&SS, 193, 29 
Hughes, J., Hartigan, P., \& Clampitt, L. 1993, AJ, 105, 571

Joergens, V., Guenther, E., Neuhäuser, R., Fernández, M., \& Vijapurkar, J. 2001, A\&A, 373, 966

Knacke, R. F., Strom, K. M., Strom, S. E., Young, E. T., \& Kunkel, W. 1973, ApJ, 179, 847

Knude, J., \& Høg, E. 1998, A\&A, 338, 897

Köhler, R. 2001, AJ, 122, 3325

König, B., Guenther, E. W., Woitas, J., \& Hatzes, A. P. 2005, A\&A, 435, 215

Lovis, C., Mayor, M., Bouchy, F., et al. 2005, A\&A, 437, 1121

Marraco, H. G., \& Rydgren, A. E. 1981, AJ, 86, 62

Mathieu, R. D. 1994, ARA\&A, 32, 465

Melo, C. H. F. 2003, A\&A, 410, 269

Montalbán, J., D’Antona, F., Kupka, F., \& Heiter, U. 2004, A\&A, 416, 1081

Murdoch, K. A., Hearnshaw, J. B., \& Clark, M. 1993, ApJ, 413, 349

Nakajima, Y., Tamura, M., Oasa, Y., \& Nakajima, T. 2000, AJ, 119, 873

Padget, D. L. 1996, ApJ, 471, 847

Palla, F., \& Stahler, S. W. 1992, ApJ, 392, 667

Palla, F., \& Stahler, S. W. 1999, ApJ, 525, 772

Prato, L., Simon, M., Mazeh, T., et al. 2002, ApJ, 569, 863

Quist, C. F., \& Lindegren, L. 2000, A\&A, 361, 770

Paulson, D. B., Saar, S. H., Cochran, W. D., \& Hatzes, A. P. 2002, AJ, 124, 572

Preibisch, T., \& Zinnecker, H. 1999, AJ, 117, 2318

Preibisch, T., Guenther, E., Zinnecker, H., et al. 1998, A\&A, 333, 619

Reipurth, B., \& Zinnecker, H. 1993, A\&A, 278, 81

Reipurth, B., Lindgren, H., Mayor, M., Mermilliod, J.-C., \& Cramer, N. 2002, $\mathrm{AJ}, 124,2813$

Saar, S. H., Butler, R. P., \& Marcy, G. W. 1998, ApJ, 498, L153

Sartori, M. J., Lepine, J. R. D., \& Dias, W. S. 2003, A\&A, 404, 913

Siess, L., Forestini, M., \& Dougados, C. 1997, A\&A, 324, 556
Silverstone, M. D., Meyer, M. R., Mamajek, E. E., et al. 2006, ApJ, 639 [arXiv: astro-ph/0511250]

Simon, M., \& Prato, L. 2004, ApJ, 613, L69

Simon, M., Dutrey, A., \& Guilloteau, S. 2000, ApJ, 545, 1034

Skrutskie, M. F., Cutri, R. M., Stiening, R., et al. 2006, AJ, 131, 1163

Song, I., Bessell, M. S., \& Zuckerman, B. 2002, A\&A, 385, 862

Stassun, K. G., Mathieu, R. D., Vaz, L. P. R., Stroud, N., \& Vrba, F. J. 2004, ApJS, 151, 357

Steffen, A. T., Mathieu, R. D., Lattanzi, M. G., et al. 2001, AJ, 122, 997

Stempels, H. C., Gahm, G. F., \& Petrov, P. P. 2007, A\&A, 461, 253

Swenson, F. J., Faulkner, J., Iglesias, C. A., Rogers, F. J., \& Alexander, D. R. 1994, ApJ, 422, L79

Takami, M., Bailey, J., \& Chrysostomou, A. 2003, A\&A, 397, 675

Teixeira, R., Ducourant, C., Sartori, M. J., et al. 2000, A\&A, 361, 1143

Torres, C. A. O., da Silva, L., Quast, G. R., de la Reza, R., \& Jilinski, E. 2000, AJ, 120, 1410

Torres, G., Guenther, E. W., Marschall, L. A., et al. 2003, AJ, 125, 825

Tout, C. A., Livio, M., \& Bonnell, I. A. 1999, MNRAS, 310, 360

Wallace, L., Hinkle, K., \& Livingston, W. 1998, An atlas of the spectrum of the solar photosphere from 13500 to $28000 \mathrm{~cm}^{-1}$ (3570 to $7405 \AA$ ) (Tucson: AZ: National Optical Astronomy Observatories)

Webb, R. A., Zuckerman, B., Platais, I., et al. 1999, ApJ, 512, L63

Weinberger, A. J., Becklin, E. E., Zuckerman, B., \& Song, I. 2004, AJ, 127, 2246

Whittet, D. C. B. 1974, MNRAS, 168, 371

Whittet, D. C. B., Prusti, T., Franco, G. A. P., et al. 1997, A\&A, 327, 1194

Wichmann, R., Bastian, U., Krautter, J., Jankovics, I., \& Ruciski, S. M. 1998, MNRAS, 301, L39

Wuchterl, G., \& Tscharnuter, W. M. 2003, A\&A, 398, 1081 
E. W. Guenther et al.: PMS spectroscopic binaries, Online Material p 1

\section{Online Material}


E. W. Guenther et al.: PMS spectroscopic binaries, Online Material p 2

Table 2. CS Cha.

\begin{tabular}{cc}
\hline \hline HJD & $R V\left[\mathrm{~km} \mathrm{~s}^{-1}\right]$ \\
\hline 2451264.54715 & $15.7 \pm 0.2$ \\
2451332.55406 & $15.5 \pm 0.1$ \\
2451621.62514 & $15.9 \pm 0.3$ \\
2452026.54612 & $14.7 \pm 0.1$ \\
2452031.51533 & $14.8 \pm 0.1$ \\
2452089.60062 & $13.5 \pm 0.6$ \\
2452093.54486 & $14.1 \pm 0.1$ \\
2452097.50960 & $14.5 \pm 0.1$ \\
2452098.55118 & $14.1 \pm 0.2$ \\
2452319.67764 & $13.0 \pm 0.3$ \\
2452322.65188 & $13.3 \pm 0.3$ \\
2452373.56573 & $12.9 \pm 0.3$ \\
2452710.51867 & $13.3 \pm 0.4$ \\
2452717.55061 & $13.2 \pm 0.3$ \\
2452723.53930 & $13.3 \pm 0.2$ \\
2453097.51064 & $13.5 \pm 0.2$ \\
2453104.56677 & $14.6 \pm 0.1$ \\
2453105.53213 & $14.2 \pm 0.2$ \\
2453122.55011 & $13.6 \pm 0.2$ \\
2453137.56055 & $13.7 \pm 0.2$ \\
2453155.57941 & $13.7 \pm 0.3$ \\
2453455.56786 & $14.6 \pm 0.1$ \\
2453168.54063 & $14.6 \pm 0.1$ \\
2453501.61418 & $14.9 \pm 0.1$ \\
2453524.53172 & $14.9 \pm 0.1$ \\
2453552.52001 & $13.5 \pm 0.1$ \\
2453601.52246 & $14.6 \pm 0.2$ \\
2453811.53842 & $15.8 \pm 0.2$ \\
2453811.58558 & $15.8 \pm 0.2$ \\
2453811.60357 & $15.8 \pm 0.2$ \\
2453813.78161 & $15.6 \pm 0.2$ \\
2453834.09873 & $14.9 \pm 0.2$ \\
\hline & \\
&
\end{tabular}

Table 3. RX J1220.6-7539.

\begin{tabular}{cr}
\hline \hline HJD & $R V\left[\mathrm{~km} \mathrm{~s}^{-1}\right]$ \\
\hline 2451262.64298 & $0.7 \pm 0.1$ \\
2451333.65495 & $-5.4 \pm 0.1$ \\
2451621.67014 & $7.9 \pm 0.1$ \\
2451737.48553 & $7.5 \pm 0.1$ \\
2452089.58864 & $1.2 \pm 0.1$ \\
2452093.53472 & $1.7 \pm 0.1$ \\
2452097.55429 & $2.0 \pm 0.1$ \\
2452098.52034 & $1.7 \pm 0.1$ \\
2452319.78210 & $8.4 \pm 0.1$ \\
2452372.63728 & $7.6 \pm 0.1$ \\
2452373.61486 & $7.2 \pm 0.1$ \\
2452384.59905 & $7.1 \pm 0.1$ \\
2452395.67658 & $6.2 \pm 0.1$ \\
2452710.65545 & $1.4 \pm 0.1$ \\
2452717.69773 & $2.2 \pm 0.1$ \\
2452723.66034 & $2.6 \pm 0.1$ \\
2453096.66843 & $0.9 \pm 0.1$ \\
2453097.71156 & $0.9 \pm 0.1$ \\
2453104.70590 & $0.4 \pm 0.1$ \\
2453105.63828 & $0.2 \pm 0.1$ \\
2453122.64088 & $-1.4 \pm 0.1$ \\
2453137.72151 & $-2.8 \pm 0.1$ \\
2453168.57480 & $-5.1 \pm 0.1$ \\
2453455.66760 & $7.8 \pm 0.1$ \\
2453522.60916 & $8.3 \pm 0.1$ \\
2453550.54048 & $7.8 \pm 0.1$ \\
2453811.55386 & $-6.1 \pm 0.1$ \\
2453813.79633 & $-6.2 \pm 0.1$ \\
2453835.16435 & $-5.6 \pm 0.1$ \\
2453849.21861 & $-4.8 \pm 0.1$ \\
\hline &
\end{tabular}

Table 5. RX J1534.1-3916.

\begin{tabular}{cc}
\hline \hline HJD & $R V\left[\mathrm{~km} \mathrm{~s}^{-1}\right]$ \\
\hline 2451261.76390 & $-25.0 \pm 0.1$ \\
2451335.77111 & $-27.5 \pm 1.0$ \\
2452093.73652 & $-12.7 \pm 1.5$ \\
2452372.74166 & $-10.6 \pm 0.1$ \\
2452384.71011 & $-10.9 \pm 0.5$ \\
2452395.72062 & $-11.4 \pm 0.4$ \\
2452710.78396 & $-12.3 \pm 0.5$ \\
2452717.78285 & $-12.0 \pm 0.2$ \\
2452723.77086 & $-11.3 \pm 0.2$ \\
2453096.73954 & $-12.4 \pm 0.7$ \\
2453104.77290 & $-11.3 \pm 0.4$ \\
2453105.73478 & $-11.5 \pm 0.5$ \\
2453122.76000 & $-13.0 \pm 0.1$ \\
2453137.77322 & $-12.6 \pm 0.4$ \\
2453168.62275 & $-11.6 \pm 0.1$ \\
2453501.73605 & $-13.7 \pm 0.1$ \\
2453523.74779 & $-13.8 \pm 0.1$ \\
2453556.77084 & $-11.3 \pm 0.2$ \\
2453556.78210 & $-11.1 \pm 0.5$ \\
2453601.55318 & $-13.6 \pm 0.3$ \\
2453603.51374 & $-10.8 \pm 0.2$ \\
2453811.68239 & $-12.0 \pm 0.2$ \\
2453813.84489 & $-12.1 \pm 0.7$ \\
2453835.23052 & $-11.9 \pm 0.3$ \\
\hline &
\end{tabular}


E. W. Guenther et al.: PMS spectroscopic binaries, Online Material p 3

Table 6. RX J1559.2-3814.

\begin{tabular}{crr}
\hline \hline HJD & $\begin{array}{r}R V\left[\mathrm{~km} \mathrm{~s}^{-1}\right] \\
\text { A component }\end{array}$ & $\begin{array}{r}R V\left[\mathrm{~km} \mathrm{~s}^{-1}\right] \\
\text { B component }\end{array}$ \\
\hline 2451264.71496 & $-6.7 \pm 0.3$ & $12.2 \pm 0.6$ \\
2451331.71643 & $-7.9 \pm 0.2$ & $12.3 \pm 1.1$ \\
2451622.78442 & $19.6 \pm 0.4$ & $-14.2 \pm 0.4$ \\
2451623.78926 & $20.6 \pm 0.8$ & $-14.6 \pm 0.6$ \\
2451625.71327 & $19.1 \pm 0.9$ & $-15.2 \pm 0.7$ \\
2453097.64088 & $4.2 \pm 0.1^{1}$ & \\
2453105.69324 & $2.6 \pm 0.4^{1}$ & \\
2453122.77331 & $0.9 \pm 0.3^{1}$ & \\
2453137.78841 & $-2.8 \pm 0.9$ & \\
2453168.81587 & $-8.1 \pm 0.4$ & $8.4 \pm 0.8$ \\
2453168.82963 & $-7.8 \pm 0.3$ & $9.1 \pm 1.0$ \\
2453501.75306 & $18.6 \pm 0.1$ & $-16.4 \pm 1.1$ \\
2453503.78331 & $19.2 \pm 0.2$ & $-15.1 \pm 0.4$ \\
2453523.76173 & $18.5 \pm 0.2$ & $-15.2 \pm 1.1$ \\
2453556.79982 & $7.7 \pm 1.6^{1}$ & \\
2453556.81105 & $8.1 \pm 1.3^{1}$ & \\
2453601.56957 & $0.6 \pm 0.4^{1}$ & \\
2453603.53176 & $-0.1 \pm 0.6^{1}$ & \\
2453811.70502 & $2.0 \pm 1.1^{1}$ & \\
2453811.72015 & $1.4 \pm 1.0^{1}$ & \\
2453813.81043 & $1.4 \pm 0.7^{1}$ & \\
2453813.82554 & $1.2 \pm 0.5^{1}$ & \\
2453838.28599 & $3.1 \pm 1.0^{1}$ & \\
\hline
\end{tabular}

${ }^{1}$ Just A-component.

Table 8. GSC 06209-00735.

\begin{tabular}{cr}
\hline \hline HJD & \multicolumn{1}{c}{$R V\left[\mathrm{~km} \mathrm{~s}^{-1}\right]$} \\
\hline 2451260.79532 & $-5.7 \pm 0.1$ \\
2451333.79536 & $-6.7 \pm 0.1$ \\
2451622.87285 & $-9.3 \pm 0.1$ \\
2451737.59186 & $-10.1 \pm 0.2$ \\
2452093.77154 & $-10.4 \pm 0.4$ \\
2452395.75208 & $-8.6 \pm 0.1$ \\
2452396.70830 & $-8.5 \pm 0.1$ \\
2452372.81128 & $-8.7 \pm 0.1$ \\
2452384.73607 & $-8.4 \pm 0.1$ \\
2452710.79456 & $-6.8 \pm 0.1$ \\
2452717.79687 & $-6.4 \pm 0.1$ \\
2452723.78469 & $-6.5 \pm 0.1$ \\
2453096.76265 & $-4.1 \pm 0.1$ \\
2453097.75000 & $-4.2 \pm 0.1$ \\
2453104.79092 & $-4.3 \pm 0.1$ \\
2453105.87514 & $-4.3 \pm 0.1$ \\
2453122.78632 & $-4.4 \pm 0.1$ \\
2453137.81404 & $-4.5 \pm 0.1$ \\
2453168.63464 & $-5.0 \pm 0.1$ \\
2453601.59793 & $-8.8 \pm 0.1$ \\
2453616.52275 & $-8.8 \pm 0.1$ \\
2453811.75370 & $-9.7 \pm 0.1$ \\
2453838.36166 & $-9.5 \pm 0.1$ \\
2453847.35257 & $-9.8 \pm 0.1$ \\
\hline
\end{tabular}

Table 10. GSC 06213-00306.

\begin{tabular}{crr}
\hline \hline HJD & $\begin{array}{r}R V\left[\mathrm{~km} \mathrm{~s}^{-1}\right] \\
\text { A component }\end{array}$ & $\begin{array}{r}R V\left[\mathrm{~km} \mathrm{~s}^{-1}\right] \\
\text { B component }\end{array}$ \\
\hline 2451333.82932 & $-5.5 \pm 0.5^{1}$ & \\
2451622.89550 & $-24.3 \pm 0.1$ & $10.9 \pm 0.3$ \\
2451623.75952 & $-24.1 \pm 0.1$ & $11.4 \pm 0.2$ \\
2451624.73672 & $-24.8 \pm 0.2$ & $11.9 \pm 0.2$ \\
2451625.73770 & $-25.3 \pm 0.3$ & $12.4 \pm 0.3$ \\
2451737.66113 & $-18.0 \pm 0.2$ & $4.1 \pm 0.5$ \\
2452089.46697 & $-9.0 \pm 0.2^{1}$ & \\
2452093.50599 & $-7.0 \pm 0.2^{1}$ & \\
2452097.59641 & $-6.9 \pm 0.1^{1}$ & \\
2452396.72380 & $4.5 \pm 0.8$ & $-18.6 \pm 0.2$ \\
2452372.76696 & $4.1 \pm 0.9$ & $-17.5 \pm 0.2$ \\
2452384.74816 & $5.3 \pm 0.4$ & $-18.6 \pm 0.1$ \\
2452385.71382 & $4.8 \pm 0.6$ & $-18.8 \pm 0.3$ \\
2452710.82314 & $4.4 \pm 0.5$ & $-18.8 \pm 0.3$ \\
2452717.82525 & $6.4 \pm 0.5$ & $-19.2 \pm 0.2$ \\
2452723.81319 & $5.5 \pm 0.5$ & $-19.1 \pm 0.2$ \\
2451260.89577 & $-6.0 \pm 0.4^{1}$ & \\
2451260.89577 & $-6.1 \pm 0.5^{1}$ & \\
2453603.57132 & $5.7 \pm 0.6$ & $-19.7 \pm 0.9$ \\
2453811.76438 & $-2.6 \pm 1.0^{2}$ & \\
2453813.87632 & $-2.8 \pm 1.0^{2}$ & \\
2453838.38271 & $-7.0 \pm 0.4^{2}$ & \\
\hline
\end{tabular}

${ }^{1}$ both components, unresolved. ${ }^{2}$ only the A-component.

Table 12. VW Cha.

\begin{tabular}{cr}
\hline \hline HJD & $R V\left[\mathrm{~km} \mathrm{~s}^{-1}\right]$ \\
\hline 2451262.57801 & $14.9 \pm 0.5$ \\
2451333.57002 & $15.4 \pm 0.2$ \\
2452031.53243 & $17.2 \pm 0.2$ \\
2452319.70304 & $19.8 \pm 1.0$ \\
2452322.67626 & $18.6 \pm 0.6$ \\
2452372.53625 & $18.4 \pm 1.2$ \\
2452384.53498 & $15.3 \pm 0.4$ \\
2452710.59595 & $16.3 \pm 0.2$ \\
2452717.59503 & $16.3 \pm 0.4$ \\
2452723.58209 & $22.0 \pm 2.4$ \\
2453096.65990 & $18.7 \pm 0.9$ \\
2453104.58111 & $15.2 \pm 0.2$ \\
2453105.55467 & $17.5 \pm 0.1$ \\
2453122.59151 & $18.4 \pm 0.5$ \\
2453137.59880 & $16.1 \pm 0.4$ \\
2453155.64238 & $14.6 \pm 2.6$ \\
2453122.59151 & $18.5 \pm 0.4$ \\
2453522.57695 & $16.4 \pm 0.1$ \\
2453524.54647 & $18.3 \pm 0.4$ \\
\hline
\end{tabular}


E. W. Guenther et al.: PMS spectroscopic binaries, Online Material p 4

Table 13. GSC 06793-00569.

\begin{tabular}{cr}
\hline \hline HJD & $R V\left[\mathrm{~km} \mathrm{~s}^{-1}\right]$ \\
\hline 2451260.90498 & $-6.5 \pm 0.2$ \\
2451333.84131 & $-4.2 \pm 0.3$ \\
2451737.67610 & $-6.5 \pm 0.2$ \\
2452089.47704 & $-6.8 \pm 0.4$ \\
2452093.64727 & $-4.5 \pm 0.4$ \\
2452097.60976 & $-8.6 \pm 0.9$ \\
2452373.71233 & $-5.7 \pm 0.2$ \\
2452385.67462 & $-5.1 \pm 0.1$ \\
2452395.83337 & $-5.8 \pm 0.1$ \\
2452396.81929 & $-5.7 \pm 0.1$ \\
2452710.86108 & $-7.8 \pm 0.1$ \\
2452717.86133 & $-6.3 \pm 0.1$ \\
2452723.82421 & $-7.0 \pm 0.1$ \\
2453097.68383 & $-5.8 \pm 0.1$ \\
2453104.81253 & $-5.2 \pm 0.1$ \\
2453105.78900 & $-6.2 \pm 0.1$ \\
2453122.83664 & $-5.5 \pm 0.1$ \\
2453137.83685 & $-6.4 \pm 0.1$ \\
2453168.70120 & $-6.9 \pm 0.1$ \\
\hline
\end{tabular}

Table 14. RX J1415.0-7822.

\begin{tabular}{cr}
\hline \hline HJD & $R V\left[\mathrm{~km} \mathrm{~s}^{-1}\right]$ \\
\hline 2451261.69317 & $19.7 \pm 0.4$ \\
2451622.67334 & $18.1 \pm 1.3$ \\
2451737.50348 & $17.1 \pm 1.7$ \\
2452019.67697 & $20.1 \pm 0.3$ \\
2452026.69069 & $19.0 \pm 0.6$ \\
2452031.67124 & $19.1 \pm 0.1$ \\
2452098.53621 & $16.2 \pm 1.0$ \\
2452372.67493 & $21.0 \pm 0.2$ \\
2452373.64078 & $19.3 \pm 0.2$ \\
2452384.64679 & $19.8 \pm 0.5$ \\
2452710.69367 & $19.2 \pm 0.3$ \\
2452717.71153 & $19.0 \pm 0.5$ \\
2452723.69779 & $18.9 \pm 0.4$ \\
2453096.86233 & $18.7 \pm 0.2$ \\
2453104.74397 & $20.3 \pm 0.5$ \\
2453105.70977 & $20.2 \pm 0.4$ \\
2453122.73806 & $18.6 \pm 0.5$ \\
2453137.75246 & $20.5 \pm 0.3$ \\
2453168.65138 & $18.7 \pm 0.3$ \\
\hline
\end{tabular}

Table 15. HK Lup.

\begin{tabular}{cr}
\hline \hline HJD & $R V\left[\mathrm{~km} \mathrm{~s}^{-1}\right]$ \\
\hline 2451261.90059 & $-1.3 \pm 0.5$ \\
2451262.69194 & $-5.0 \pm 0.5$ \\
2451622.81043 & $-5.3 \pm 0.7$ \\
2451733.68220 & $-2.5 \pm 0.2$ \\
2452026.83013 & $-5.2 \pm 0.7$ \\
2452031.79988 & $-5.7 \pm 1.2$ \\
2452093.75963 & $-1.4 \pm 0.4$ \\
2452098.59938 & $-1.9 \pm 0.3$ \\
2452395.79978 & $0.2 \pm 0.5$ \\
2452396.77110 & $4.5 \pm 0.2$ \\
2452372.75478 & $-0.8 \pm 0.4$ \\
2452384.72338 & $-2.3 \pm 0.1$ \\
2452385.69702 & $-0.6 \pm 0.1$ \\
2452710.80837 & $-0.6 \pm 0.4$ \\
2452717.81062 & $-2.3 \pm 0.1$ \\
2452723.79847 & $-0.4 \pm 0.1$ \\
2453096.77689 & $-1.8 \pm 0.3$ \\
2453104.80132 & $-2.3 \pm 2.9$ \\
2453105.76881 & $-8.6 \pm 0.4$ \\
2453122.81946 & $-8.7 \pm 0.2$ \\
2453455.75212 & $1.6 \pm 0.8$ \\
\hline
\end{tabular}

Table 16. GSC 06793-01406.

\begin{tabular}{cr}
\hline \hline HJD & $R V\left[\mathrm{~km} \mathrm{~s}^{-1}\right]$ \\
\hline 2451625.80168 & $-6.9 \pm 0.6$ \\
2451737.70415 & $-7.4 \pm 0.6$ \\
2452089.73677 & $-7.3 \pm 0.4$ \\
2452093.78125 & $-5.8 \pm 0.7$ \\
2452384.81131 & $-5.5 \pm 0.1$ \\
2452717.88212 & $-7.9 \pm 0.2$ \\
2452723.87107 & $-6.8 \pm 0.1$ \\
2453096.84966 & $-5.7 \pm 0.3$ \\
2453104.85577 & $-5.6 \pm 0.1$ \\
2453105.83868 & $-7.1 \pm 0.1$ \\
2453122.87716 & $-7.0 \pm 0.2$ \\
2453137.88922 & $-8.0 \pm 0.2$ \\
2453168.74239 & $-6.2 \pm 0.1$ \\
\hline
\end{tabular}

Table 17. GSC 06793-00994.

\begin{tabular}{cc}
\hline \hline HJD & $R V\left[\mathrm{~km} \mathrm{~s}^{-1}\right]$ \\
\hline 2451262.70144 & $-2.8 \pm 0.1$ \\
2451737.69110 & $-4.2 \pm 0.5$ \\
2452089.51975 & $-3.9 \pm 0.1$ \\
2452093.68633 & $-2.6 \pm 0.3$ \\
2452396.80296 & $-0.9 \pm 0.1$ \\
2452372.82468 & $-0.7 \pm 0.2$ \\
2452373.69694 & $-4.4 \pm 0.3$ \\
2452384.79676 & $-0.9 \pm 0.1$ \\
2452385.73404 & $-5.0 \pm 0.1$ \\
2452710.87369 & $-5.4 \pm 0.1$ \\
2452717.83773 & $-3.4 \pm 0.1$ \\
2452723.83505 & $-3.6 \pm 0.1$ \\
2453096.79173 & $-2.2 \pm 0.1$ \\
2453104.83363 & $-3.6 \pm 0.1$ \\
2453105.80278 & $-2.5 \pm 0.1$ \\
2453122.84741 & $-3.6 \pm 0.1$ \\
2453137.84760 & $-3.0 \pm 0.1$ \\
2453168.72226 & $-3.0 \pm 0.1$ \\
\hline
\end{tabular}


Table 18. The single stars: Chamaeleon.

\begin{tabular}{|c|c|c|c|c|c|c|c|c|c|c|}
\hline & Region & $\begin{array}{c}E W \mathrm{H} \alpha^{1} \\
{[\AA ̊}\end{array}$ & $\begin{array}{c}E W \text { LiI } \\
{[\AA]}\end{array}$ & $\begin{array}{l}\text { Spec } \\
\text { type }\end{array}$ & $\begin{array}{c}m_{K} \\
{[\mathrm{mag}]}\end{array}$ & $\begin{array}{c}\text { RA } \\
(2000.0)\end{array}$ & $\begin{array}{c}\text { Dec } \\
(2000.0)\end{array}$ & $\begin{array}{r}R V \\
{\left[\mathrm{~km} \mathrm{~s}^{-1}\right]}\end{array}$ & $\begin{array}{r}\sigma R V \\
{\left[\mathrm{~km} \mathrm{~s}^{-1}\right]}\end{array}$ & $\begin{array}{l}\text { No. of } \\
\text { spectra }\end{array}$ \\
\hline EG Cha & Cha & $-1.1 \pm 0.1$ & $0.53 \pm 0.01$ & $\mathrm{~K} 7$ & $7.338 \pm 0.021$ & 083656.2 & -785646 & $17.4 \pm 0.2$ & 0.4 & $\frac{1}{3}$ \\
\hline EO Cha & Cha & $-1.0 \pm 0.2$ & $0.53 \pm 0.04$ & M0 & $8.732 \pm 0.021$ & 084431.9 & -784631 & $17.1 \pm 0.3$ & 0.5 & 2 \\
\hline EQ $\mathrm{Cha}^{3}$ & Cha & $-6.5 \pm 1.1$ & $0.57 \pm 0.04$ & M3 & $8.410 \pm 0.031$ & 084759.2 & -785458 & $17.9 \pm 1.0$ & 2.7 & 8 \\
\hline RX J0850.1-7554 & Cha & abs & $0.27 \pm 0.01$ & G5 & $8.704 \pm 0.019$ & 085005.4 & -755438 & $18.1 \pm 1.0$ & 1.3 & 2 \\
\hline RX J0915.5-7609 & Cha & $-1.1 \pm 0.2$ & $0.55 \pm 0.03$ & K6 & $8.488 \pm 0.033$ & 091529.1 & -760847 & $20.3 \pm 0.1$ & 0.1 & 3 \\
\hline RX J0917.2-7744 & Cha & abs & $0.33 \pm 0.06$ & G2 & $8.812 \pm 0.023$ & 091711.4 & -774415 & $5.7 \pm 1.8$ & 3.2 & 3 \\
\hline HD84075 & Cha & abs & $0.17 \pm 0.01$ & G2 & $7.160 \pm 0.016$ & 093617.8 & -782042 & $5.2 \pm 0.1$ & 0.1 & 3 \\
\hline HD86356 & Cha & fi & $0.34 \pm 0.05$ & G8 & $8.040 \pm 0.029$ & 095150.7 & -790138 & $13.2 \pm 0.4$ & 0.5 & 2 \\
\hline HD86588 & Cha & abs & $0.11 \pm 0.01$ & F6 & $7.994 \pm 0.029$ & 095313.7 & -793328 & $2.4 \pm 0.5$ & 2.4 & 3 \\
\hline RXJ1001.1-7913 & Cha & $-2.5 \pm 0.7$ & $0.10 \pm 0.01$ & K8 & $9.216 \pm 0.021$ & 100108.8 & -791308 & $14.4 \pm 0.4$ & 0.6 & 2 \\
\hline RXJ1005.3-7749 & Cha & $-4.2 \pm 0.1$ & $0.58 \pm 0.01$ & M1 & $8.892 \pm 0.019$ & 100517.6 & -774906 & $16.3 \pm 0.2$ & 0.3 & 2 \\
\hline SX Cha $E^{4}$ & Cha & $-20 \pm 5$ & $0.65 \pm 0.04$ & M0 & $8.685 \pm 0.024$ & 105559.8 & -772440 & $13.9 \pm 0.5$ & 1.1 & 5 \\
\hline SY Cha & Cha & $-13 \pm 2$ & $0.56 \pm 0.03$ & M0 & $8.631 \pm 0.019$ & 105630.5 & -771139 & $14.0 \pm 0.3$ & 0.5 & 3 \\
\hline TW Cha & Cha & $-28 \pm 11$ & $0.41 \pm 0.01$ & K7 & $8.616 \pm 0.021$ & 105901.1 & -772241 & $17.8 \pm 0.1$ & 0.1 & 2 \\
\hline CR Cha (=Sz6) & Cha & $-34 \pm 2$ & $0.43 \pm 0.04$ & K2 & $7.310 \pm 0.023$ & 105907.0 & -770140 & $16.7 \pm 1.1$ & 2.0 & 3 \\
\hline CT Cha & Cha & $-40 \pm 21$ & $0.40 \pm 0.05$ & K7 & $8.661 \pm 0.021$ & 110409.1 & -762719 & $15.2 \pm 0.3$ & 0.9 & 13 \\
\hline DI Cha ${ }^{5}$ & Cha & $-17 \pm 1$ & $0.26 \pm 0.01$ & $\mathrm{G} 2$ & $6.217 \pm 0.020$ & 110720.7 & -773807 & $14.6 \pm 0.2$ & 0.3 & 2 \\
\hline VW Cha ${ }^{6}$ & Cha & $-57 \pm 11$ & $0.44 \pm 0.05$ & M0.5 & $6.962 \pm 0.026$ & 110801.8 & -774229 & $17.2 \pm 0.5$ & 2.0 & 17 \\
\hline WW Cha ${ }^{2}$ & Cha & $-57 \pm 13$ & $0.3 \pm 0.2$ & K5 & $6.083 \pm 0.053$ & 111000.7 & -763459 & $15.0 \pm 2.0$ & 0.5 & 2 \\
\hline HBC 584 & Cha & $-77 \pm 1$ & $0.3 \pm 0.1$ & K7 & $9.175 \pm 0.024$ & 111049.6 & -771752 & $13.6 \pm 0.1$ & 0.2 & 2 \\
\hline $\mathrm{Sz} 41^{7}$ & Cha & $-2.0 \pm 0.9$ & $0.43 \pm 0.02$ & K0 & $7.999 \pm 0.031$ & 111224.5 & -763706 & $14.6 \pm 0.3$ & 1.1 & 18 \\
\hline VZ Cha ${ }^{2}$ & Cha & $-32 \pm 4$ & $0.31 \pm 0.01$ & K6 & $8.242 \pm 0.038$ & 110923.8 & -762321 & $16.3 \pm 0.4$ & 0.6 & 2 \\
\hline $\mathrm{CV} \mathrm{Cha}^{2}, 5$ & Cha & $-67 \pm 16$ & $0.34 \pm 0.02$ & G8 & $6.845 \pm 0.026$ & 111227.7 & -764422 & $16.1 \pm 1.2$ & 1.8 & 2 \\
\hline GSC 07739-02180 & $\mathrm{SC}$ & $-3.9 \pm 1.2$ & $0.52 \pm 0.01$ & & $8.053 \pm 0.029$ & 112105.5 & -384517 & $12.1 \pm 0.7$ & 1.2 & 3 \\
\hline RXJ1129.2-7546 & Cha & fi & $0.49 \pm 0.02$ & K3 & $8.878 \pm 0.024$ & 112912.7 & -754626 & $14.6 \pm 0.5$ & 0.8 & 3 \\
\hline RXJ1140.3-8321 & Cha & $-0.3 \pm 0.2$ & $0.21 \pm 0.01$ & K2 & $8.635 \pm 0.019$ & 114016.6 & -832100 & $12.7 \pm 0.1$ & 0.1 & 3 \\
\hline RXJ1150.4-7704 & Cha & $-1.3 \pm 0.5$ & $0.40 \pm 0.06$ & $\mathrm{~K} 2$ & $8.970 \pm 0.021$ & 115028.9 & -770438 & $-3.3 \pm 1.0$ & 2.1 & 4 \\
\hline T Cha & Cha & $-8.8 \pm 9.9$ & $0.41 \pm 0.03$ & G8 & $6.954 \pm$ & 115713.5 & -792132 & $14.0 \pm 1.3$ & 5.3 & 17 \\
\hline RXJ1159.7-7601 & Cha & $-0.2 \pm 0.1$ & $0.46 \pm 0.01$ & K2 & $8.304 \pm 0.027$ & 115942.3 & -760126 & $13.8 \pm 0.1$ & 0.1 & 3 \\
\hline RX J1202.1-7853 & Cha & $-3.3 \pm 0.5$ & $0.56 \pm 0.02$ & K7 & $8.307 \pm 0.023$ & 120203.8 & -785301 & $17.1 \pm 0.2$ & 0.3 & 3 \\
\hline HD106772 & Cha & abs & $0.21 \pm 0.01$ & G2 & $6.231 \pm 0.031$ & 121726.9 & -803506 & $-12.9 \pm 0.2$ & 0.3 & 2 \\
\hline RXJ1219.7-7403 & Cha & $-4.5 \pm 1.6$ & $0.56 \pm 0.01$ & K8 & $8.858 \pm 0.023$ & 121943.8 & -740357 & $13.8 \pm 0.1$ & 0.2 & 2 \\
\hline RXJ1220.4-7407 & Cha & $-2.9 \pm 0.6$ & $0.61 \pm 0.01$ & K7 & $8.367 \pm 0.022$ & 122021.9 & -740739 & $12.3 \pm 0.4$ & 0.6 & 2 \\
\hline HD107722 & Cha & abs & $0.08 \pm 0.01$ & F6 & $7.135 \pm 0.034$ & 122329.0 & -774051 & $13.4 \pm 0.4$ & 0.6 & 2 \\
\hline RXJ1233.5-7523 & Cha & abs & $0.14 \pm 0.01$ & K0 & $7.756 \pm 0.040$ & 123332.0 & -752325 & $15.5 \pm 0.1$ & 0.1 & 2 \\
\hline RXJ1239.4-7502 & Cha & abs & $0.41 \pm 0.01$ & K2 & $7.777 \pm 0.021$ & 123921.3 & -750239 & $13.9 \pm 0.2$ & 0.3 & 2 \\
\hline BC Cha & Cha & $-72 \pm 18$ & $0.52 \pm 0.03$ & & $9.354 \pm 0.021$ & 130159.0 & -775122 & $14.3 \pm 0.2$ & 0.4 & 2 \\
\hline RX J1325.7-7955 & Cha & abs & $0.10 \pm 0.05$ & K1 & $8.932 \pm 0.022$ & 132542.8 & -795525 & $-8.4 \pm 3.6$ & 7.1 & 4 \\
\hline CPD-75 902 & Cha & abs & $0.22 \pm 0.01$ & K0 & $8.015 \pm 0.053$ & 134912.9 & -754948 & $-0.8 \pm 0.1$ & 0.2 & 2 \\
\hline RXJ1415.0-7822 & Cha & fi & $0.26 \pm 0.05$ & G5 & $9.876 \pm 0.023$ & 141501.8 & $\begin{array}{lll}-78 & 22 & 12\end{array}$ & $-3.1 \pm 0.3$ & 1.3 & 18 \\
\hline
\end{tabular}

${ }^{1} \mathrm{em}=$ emission, abs = absorption fi = filled in; ${ }^{2}$ WW Cha, VZ Cha, CV Cha: also observed by Melo et al. (2003) and found to be single; ${ }^{3}$ EQ Cha: difficult object, cc-function asymmetric, unlikely to be binary; ${ }^{4} \mathrm{SX}$ Cha: visual binary M0 primary and M3 secondary with a separation of 2'. 2. (Reipurth \& Zinnecker 1993), only eastern component observed; ${ }^{5}$ DI Cha (LkH $\alpha$ 332-17): binary with separation of 4.' $9 \pm 0 .{ }^{\prime \prime} 2$ (Ghez et al. 1997); ${ }^{6}$ VW Cha: triple system, separation of $0.66 \pm 0.03$ and 17'. $\pm 22^{\prime \prime}$ (Ghez et al. 1997); ${ }^{7} \mathrm{Sz} 41$ : triple system, separation of 1'.5 \pm 0. and $^{\prime \prime}$ 12 '. $4 \pm$ 0.' 6 (Ghez et al. 1997). 
Table 19. The single stars: Lupus, Scorpius Centaurus.

\begin{tabular}{|c|c|c|c|c|c|c|c|c|c|c|}
\hline & Region & $\begin{array}{c}E W \mathrm{H} \alpha^{1} \\
{[\AA]}\end{array}$ & $\begin{array}{c}E W \text { LiI } \\
{[\AA]}\end{array}$ & $\begin{array}{l}\text { Spec } \\
\text { type }\end{array}$ & $\begin{array}{c}m_{K} \\
{[\mathrm{mag}]}\end{array}$ & $\begin{array}{c}\text { RA } \\
(2000.0)\end{array}$ & $\begin{array}{c}\text { Dec } \\
(2000.0)\end{array}$ & $\begin{array}{r}R V \\
{\left[\mathrm{~km} \mathrm{~s}^{-1}\right]}\end{array}$ & $\begin{array}{r}\sigma R V \\
{\left[\mathrm{~km} \mathrm{~s}^{-1}\right]}\end{array}$ & $\begin{array}{l}\text { No. of } \\
\text { spectra }\end{array}$ \\
\hline LQ Lup & Lup & $-1.5 \pm 0.5$ & $0.26 \pm 0.03$ & G8 & $8.809 \pm 0.021$ & 150837.8 & -442317 & $7.6 \pm 1.5$ & 5.8 & 17 \\
\hline LS Lup & Lup & $-0.7 \pm 0.1$ & $0.47 \pm 0.01$ & K1 & $9.454 \pm 0.021$ & 151552.8 & -441816 & $6.8 \pm 0.4$ & 0.5 & 2 \\
\hline RX J1516.6-4406 & Lup & fi & $0.54 \pm 0.13$ & $\mathrm{~K} 2$ & $9.193 \pm 0.019$ & 151636.8 & -440719 & $4.4 \pm 1.3$ & 5.5 & 18 \\
\hline MM Lup & Lup & fi & $0.47 \pm 0.01$ & & $9.260 \pm 0.023$ & 152325.7 & -405545 & $4.4 \pm 0.1$ & 0.2 & 3 \\
\hline MN Lup ${ }^{3}$ & Lup & $-6.1 \pm 0.8$ & $0.44 \pm 0.05$ & M2 & $9.496 \pm 0.019$ & 152330.4 & -382129 & $6.6 \pm 3.4$ & 6.9 & 4 \\
\hline MP Lup & Lup & $\mathrm{fi}^{1}$ & $0.36 \pm 0.01$ & K1 & $8.930 \pm 0.019$ & 152432.4 & -365202 & $3.3 \pm 0.3$ & 0.6 & 4 \\
\hline MQ Lup & Lup & $-0.5 \pm 0.2$ & $0.39 \pm 0.03$ & $\mathrm{~K} 2$ & $8.842 \pm 0.021$ & 152533.2 & -361346 & $3.6 \pm 0.1$ & 0.1 & 2 \\
\hline MR Lup & Lup & $-1.7 \pm 0.2$ & $0.47 \pm 0.03$ & K6 & $8.963 \pm 0.019$ & 152536.7 & -353732 & $-1.0 \pm 1.9$ & 4.2 & 5 \\
\hline HIP75836 & & abs & $0.04 \pm 0.01$ & K0 & $6.760 \pm 0.020$ & 152926.9 & -285052 & $-19.5 \pm 0.1$ & 0.1 & 3 \\
\hline MT Lup & Lup & $-1.3 \pm 0.5$ & $0.48 \pm 0.01$ & K5 & $9.376 \pm 0.024$ & 153802.7 & -380722 & $3.0 \pm 0.2$ & 0.3 & 2 \\
\hline RX J1538.6-3916 & Lup & abs & $0.39 \pm 0.01$ & K4 & $8.854 \pm 0.023$ & 153838.3 & -391654 & $2.5 \pm 0.2$ & 0.4 & 3 \\
\hline RX J1539.2-4455 & Lup & $-1.8 \pm 0.4$ & $0.17 \pm 0.03$ & & $9.989 \pm 0.021$ & 153912.0 & -445529 & $5.5 \pm 0.1$ & 0.1 & 2 \\
\hline MU Lup & Lup & $-0.8 \pm 0.1$ & $0.49 \pm 0.01$ & K6 & $9.187 \pm 0.026$ & 154041.2 & -375618 & $2.2 \pm 0.1$ & 0.2 & 2 \\
\hline GSC 06785-00476 & $\mathrm{SC}$ & abs & $0.30 \pm 0.01$ & G7 & $8.920 \pm 0.023$ & 154106.8 & -265626 & $-2.0 \pm 0.1$ & 0.2 & 3 \\
\hline GSC 06781-010465 & $\mathrm{SC}$ & abs & $\leq 0.2$ & G5 & $8.177 \pm 0.020$ & 154249.9 & -253641 & SB2? & - & 2 \\
\hline GW Lup ${ }^{2}$ & Lup & $-52.8 \pm 6.8$ & $0.48 \pm 0.04$ & M0 & $8.630 \pm 0.021$ & 154644.7 & -343036 & $-3.5 \pm 0.5$ & 0.8 & 2 \\
\hline HBC $603^{6}$ & Lup & $-10.4 \pm 2.2$ & $0.58 \pm 0.01$ & M0 & $8.271 \pm 0.026$ & 155147.0 & -355643 & $-2.6 \pm 0.1$ & 0.2 & 3 \\
\hline RXJ1555.4-3338 & Lup & $-0.7 \pm 0.5$ & $0.44 \pm 0.01$ & K5 & $9.353 \pm 0.022$ & 155526.3 & -333822 & $0.0 \pm 0.1$ & 0.2 & 3 \\
\hline HD142987 & $\mathrm{SC}$ & $-2.1 \pm 0.1$ & $\sim 0.3$ & G3 & $7.614 \pm 0.021$ & 155820.6 & -183725 & $6.0 \pm 1.5$ & 2.2 & 2 \\
\hline GSC 06191-00552 & $\mathrm{SC}$ & $-0.6 \pm 0.1$ & $0.48 \pm 0.01$ & K3 & $8.325 \pm 0.024$ & 155847.8 & -175759 & $-6.4 \pm 0.5$ & 0.9 & 3 \\
\hline GSC 06195-00768 & $\mathrm{SC}$ & $-0.5 \pm 0.2$ & $0.46 \pm 0.02$ & K7 & $8.372 \pm 0.025$ & 155702.4 & -195041 & $-5.1 \pm 0.2$ & 0.5 & 4 \\
\hline НВС 609 & Lup & $-30 \pm 1.0$ & $0.39 \pm 0.06$ & K8 & $8.608 \pm 0.023$ & 155916.5 & -415709 & $0.2 \pm 0.7$ & 1.0 & 2 \\
\hline RY Lup & Lup & $-3.2 \pm 0.3$ & $0.38 \pm 0.01$ & G0 & $6.976 \pm 0.019$ & 155928.4 & -402151 & $-0.4 \pm 0.5$ & 0.8 & 2 \\
\hline MZ Lup & Lup & abs & $0.33 \pm 0.02$ & G8 & $8.528 \pm 0.030$ & 160109.0 & -332014 & $1.8 \pm 0.2$ & 0.3 & 2 \\
\hline GSC 0620 & $\mathrm{SC}$ & $-0.1 \pm 0.1$ & $0.47 \pm 0.01$ & K4 & $8.727 \pm 0.025$ & 160302.7 & -180604 & $-4.9 \pm 0.1$ & 0.1 & 2 \\
\hline GSC 0678 & $\mathrm{SC}$ & $-0.1 \pm 0.1$ & $0.37 \pm 0.01$ & G7 & $8.461 \pm 0.023$ & 160550.5 & -253312 & $-4.2 \pm 0.1$ & 0.2 & 2 \\
\hline EX Lup $^{2}$ & Lup & $-24 \pm 8$ & $0.36 \pm 0.08$ & M0 & $8.496 \pm 0.021$ & 160305.5 & -401825 & $-0.3 \pm 0.1$ & 0.3 & 3 \\
\hline НBC 613 & Lup & $-32 \pm 2$ & $0.46 \pm 0.02$ & K8 & $8.724 \pm 0.023$ & 160710.0 & -391103 & $-0.5 \pm 0.3$ & 0.5 & 2 \\
\hline NQ Lup & Lup & $-1.4 \pm 0.1$ & $0.51 \pm 0.03$ & K7 & $8.714 \pm 0.021$ & 160818.3 & -384404 & $0.8 \pm 0.1$ & 0.1 & 2 \\
\hline GSC 06784-00039 & $\mathrm{SC}$ & fi & $0.37 \pm 0.01$ & G7 & $7.908 \pm 0.016$ & 160843.4 & -260217 & $-4.6 \pm 0.5$ & 0.7 & 2 \\
\hline HK Lup ${ }^{2}$ & Lup & $-22 \pm 17$ & $0.54 \pm 0.03$ & K8 & $8.014 \pm 0.021$ & 160822.5 & -390446 & $-2.2 \pm 0.8$ & 2.8 & 21 \\
\hline V1094 Sco & Lup & $-8.2 \pm 3.3$ & $0.49 \pm 0.02$ & K6 & $8.658 \pm 0.021$ & 160836.2 & -392303 & $0.4 \pm 0.3$ & 1.0 & 9 \\
\hline GSC 06793-00868 & $\mathrm{SC}$ & $-2.8 \pm 0.2$ & $0.55 \pm 0.01$ & M1 & $8.815 \pm 0.034$ & 161156.4 & -230404 & $-6.0 \pm 0.8$ & 1.3 & 3 \\
\hline GSC 06793-00797 & $\mathrm{SC}$ & $-0.7 \pm 0.2$ & $0.52 \pm 0.01$ & K4 & $8.455 \pm 0.027$ & 161302.8 & -225743 & $-6.5 \pm 0.4$ & 0.8 & 4 \\
\hline GSC 06793-005697 & $\mathrm{SC}$ & $-0.4 \pm 0.2$ & $0.47 \pm 0.20$ & K1 & $8.494 \pm 0.019$ & 161329.3 & -231106 & $-6.1 \pm 0.2$ & 1.0 & 19 \\
\hline GSC 06793-00994 & $\mathrm{SC}$ & $-0.3 \pm 0.2$ & $0.38 \pm 0.01$ & G4 & $8.608 \pm 0.023$ & 161402.1 & -230101 & $-3.1 \pm 0.3$ & 1.3 & 18 \\
\hline GSC 06801-00186 & $\mathrm{SC}$ & abs & $0.35 \pm 0.02$ & G5 & $8.686 \pm 0.022$ & 161459.3 & -275022 & $-1.7 \pm 0.1$ & 0.3 & 4 \\
\hline GSC 06793-01406 & $\mathrm{SC}$ & $-0.6 \pm 0.2$ & $0.36 \pm 0.01$ & G7 & $8.102 \pm 0.018$ & 161618.0 & -233947 & $-6.7 \pm 0.2$ & 0.8 & 13 \\
\hline GSC 06214-02384 & $\mathrm{SC}$ & fi & $0.41 \pm 0.01$ & K0 & $8.509 \pm 0.019$ & 161934.0 & -222829 & $-3.5 \pm 0.1$ & 0.1 & 3 \\
\hline GSC 06794-00156 & $\mathrm{SC}$ & $-0.3 \pm 0.1$ & $0.34 \pm 0.01$ & G6 & $7.084 \pm 0.018$ & 162451.4 & -223932 & $-3.7 \pm 0.7$ & 1.3 & 4 \\
\hline GSC 06794-00537 & $\mathrm{SC}$ & fi & $0.48 \pm 0.02$ & $\mathrm{~K} 2$ & $8.184 \pm 0.024$ & 162307.8 & -230059 & $-6.5 \pm 0.6$ & 1.0 & 3 \\
\hline GSC 06798-00035 & $\mathrm{SC}$ & fi & $0.35 \pm 0.02$ & G1 & $7.695 \pm 0.023$ & 162332.3 & -252348 & $-7.1 \pm 0.3$ & 0.2 & 2 \\
\hline GSC 06794-00337 & $\mathrm{SC}$ & fi & $0.44 \pm 0.01$ & K1 & $8.084 \pm 0.026$ & 162739.6 & -224522 & $-6.1 \pm 0.1$ & 0.1 & 4 \\
\hline
\end{tabular}

${ }^{1} \mathrm{em}=$ emission, abs = absorption fi = filled in; ${ }^{2} \mathrm{GW}$ Lup, EX Lup, HK Lup: also observed by Melo et al. (2003) and found to be single; ${ }^{3}$ MN Lup: large $v$ sin $i$ possibly a spectroscopic binary but data insufficient; ${ }^{4}$ HIP75836: listed in Simbad as eclipsing binary but no $R V$-variations observed, unlikely to be member of Lup; ${ }^{5}$ GSC 06781-01046: this object might be a short-period SB2; ${ }^{6}$ HBC 603 (Sz77): binary with separation of 1'. $8 \pm 0$ 0.' 1 (Ghez et al. 1997); ${ }^{7}$ GSC 06793-00569: asymmetric cross-correlation function, could be a visual binary. 
Table 20. The single stars: $\rho$ Ophiuchi, Corona Australis.

\begin{tabular}{|c|c|c|c|c|c|c|c|c|c|c|}
\hline & Region & $\begin{array}{c}E W \mathrm{H} \alpha^{1} \\
[\AA]]\end{array}$ & $\begin{array}{c}\text { EW LiI } \\
[\AA]]\end{array}$ & $\begin{array}{l}\text { spec } \\
\text { type }\end{array}$ & $\begin{array}{c}m_{K} \\
{[\mathrm{mag}]}\end{array}$ & $\begin{array}{c}\text { RA } \\
(2000.0)\end{array}$ & $\begin{array}{c}\text { Dec } \\
(2000.0)\end{array}$ & $\begin{array}{r}R V \\
{\left[\mathrm{~km} \mathrm{~s}^{-1}\right]}\end{array}$ & $\begin{array}{r}\sigma R V \\
{\left[\mathrm{~km} \mathrm{~s}^{-1}\right]}\end{array}$ & $\begin{array}{l}\text { No. of } \\
\text { spectra }\end{array}$ \\
\hline RXJ1612.3-1909 & Oph & $-3.9 \pm 0.1$ & $\leq 1.0$ & M2.5 & $9.605 \pm 0.019$ & 161220.9 & -190904 & $-1.6 \pm 1.6$ & 2.2 & 2 \\
\hline V1002 Sco & Oph & fi & $0.48 \pm 0.05$ & K0 & $7.494 \pm 0.026$ & 161240.5 & -185928 & $-3.7 \pm 1.0$ & 1.4 & 2 \\
\hline V2503 $\mathrm{Oph}^{2,3}$ & Oph & $-57 \pm 1$ & $0.50 \pm 0.03$ & K6-7 & $7.509 \pm 0.031$ & 162510.5 & -231914 & $-7.1 \pm 0.4$ & 0.6 & 2 \\
\hline RXJ1625.3-2402 & Oph & f1 & $0.52 \pm 0.12$ & K5 & $8.764 \pm 0.025$ & 162522.4 & -240206 & $-4.8 \pm 0.3$ & 0.4 & 2 \\
\hline V2058 Oph & Oph & $-91 \pm 12$ & $0.45 \pm 0.04$ & K5 & $7.518 \pm 0.024$ & 162556.2 & -242048 & $-4.9 \pm 0.3$ & 0.7 & 3 \\
\hline V2129 $\mathrm{Oph}^{2,4}$ & Oph & $-22 \pm 4$ & $0.53 \pm 0.02$ & K3.5 & $7.207 \pm 0.023$ & 162740.3 & -242203 & $-6.8 \pm 0.6$ & 1.7 & 8 \\
\hline HBC $268^{2}$ & Oph & $-42 \pm 2$ & $0.44 \pm 0.02$ & $\mathrm{~K} 2-3$ & $7.610 \pm 0.024$ & 163133.5 & -242737 & $-6.3 \pm 0.5$ & 0.9 & 4 \\
\hline HD173148 5 & $\mathrm{CrA}$ & abs & $0.27 \pm 0.03$ & G5 & $7.204 \pm 0.020$ & 184534.8 & -375020 & $0.1 \pm 0.4$ & 1.6 & 17 \\
\hline $\mathrm{S} \mathrm{CrA}^{2,6}$ & $\mathrm{CrA}$ & $-51 \pm 6$ & $0.20 \pm 0.01$ & K6 & $6.107 \pm 0.021$ & 190108.6 & -365720 & $0.9 \pm 0.9$ & 1.6 & 3 \\
\hline V709 CrA & $\mathrm{CrA}$ & fi & $0.40 \pm 0.02$ & K0 & $7.713 \pm 0.021$ & 190134.9 & -370057 & $-1.3 \pm 0.3$ & 0.7 & 6 \\
\hline DG & $\mathrm{CrA}$ & $-53 \pm 16$ & $0.36 \pm 0.03$ & & $7.952 \pm 0.026$ & 190155.2 & -372341 & $-1.8 \pm 0.4$ & 0.9 & 6 \\
\hline V702 CrA & $\mathrm{CrA}$ & abs,fi & $0.32 \pm 0.01$ & G5 & $8.354 \pm 0.027$ & 190202.0 & -370744 & $-0.7 \pm 0.1$ & 0.5 & 19 \\
\hline $\mathrm{HBC}$ & $\mathrm{CrA}$ & fi & $0.48 \pm 0.03$ & K5 & $9.229 \pm 0.030$ & 190222.1 & -365541 & $-0.1 \pm 0.3$ & 0.5 & 3 \\
\hline $\mathrm{Kn} \mathrm{H} \alpha 14^{7}$ & $\mathrm{CrA}$ & $-27 \pm 13$ & $0.53 \pm 0.08$ & M0 & $8.446 \pm 0.017$ & 190233.1 & -365821 & $10.5 \pm 4.4$ & 12 & 9 \\
\hline
\end{tabular}

${ }^{1} \mathrm{em}=$ emission, abs = absorption fi = filled in; ${ }^{2}$ V2503 Oph (Haro 1-4), V2129 Oph (SR 9), HBC 268 (Haro 1-16), S CrA also observed by Melo et al. (2003) and found to be single; ${ }^{3}$ V2503 Oph (Haro 1-4): binary with separation of 0.'72 (Ghez et al. 1997); ${ }^{4}$ V2129 Oph (SR 9): binary with separation of 0.'59 (Ghez et al. 1997); ${ }^{5}$ HD173148: binary with separation of 1.'1; ${ }^{6} \mathrm{~S}$ CrA: binary with separation of 1".41 \pm 0'06 (Ghez et al. 1997); ${ }^{7} \mathrm{Kn} \mathrm{H} \alpha 14$ : this might be a spectroscopic binary. 